\title{
REVIEW ARTICLE OPEN Fit-for-purpose block polymer membranes molecularly engineered for water treatment
}

\author{
Yizhou Zhang ${ }^{1}$, Noelia E. Almodovar-Arbelo ${ }^{2}$, Jacob L. Weidman ${ }^{1}$, David S. Corti ${ }^{2}$, Bryan W. Boudouris (D) $^{2,3}$ and William A. Phillip ${ }^{1}$
}

Continued stresses on fresh water supplies necessitate the utilization of non-traditional resources to meet the growing global water demand. Desalination and hybrid membrane processes are capable of treating non-traditional water sources to the levels demanded by users. Specifically, desalination can produce potable water from seawater, and hybrid processes have the potential to recover valuable resources from wastewater while producing water of a sufficient quality for target applications. Despite the demonstrated successes of these processes, state-of-the-art membranes suffer from limitations that hinder the widespread adoption of these water treatment technologies. In this review, we discuss nanoporous membranes derived from self-assembled block polymer precursors for the purposes of water treatment. Due to their well-defined nanostructures, myriad chemical functionalities, and the ability to molecularly-engineer these properties rationally, block polymer membranes have the potential to advance water treatment technologies. We focus on block polymer-based efforts to: (1) nanomanufacture large areas of highperformance membranes; (2) reduce the characteristic pore size and push membranes into the reverse osmosis regime; and (3) design and implement multifunctional pore wall chemistries that enable solute-specific separations based on steric, electrostatic, and chemical affinity interactions. The use of molecular dynamics simulations to guide block polymer membrane design is also discussed because its ability to systematically examine the available design space is critical for rapidly translating fundamental understanding to water treatment applications. Thus, we offer a full review regarding the computational and experimental approaches taken in this arena to date while also providing insights into the future outlook of this emerging technology.

npj Clean Water (2018)1:2; doi:10.1038/s41545-018-0002-1

\section{INTRODUCTION}

The growing global population and climate change have stressed fresh water supplies to critical levels. Simultaneously, human activity has degraded the quality of these vital resources through the discharge of chemicals into ground and surface waters, ${ }^{1-3}$ while the deterioration of centralized water distribution systems hinders conservation of these resources. ${ }^{4-6}$ As pressure from these factors mounts, a recognition has emerged that, along with better conservation and management of existing resources, nontraditional sources of water (e.g., seawater, brackish water, and wastewater) will need to become components of the water supply portfolio. Membrane separations have already made an impact in the water treatment arena as highlighted by the successful implementation of seawater desalination by reverse osmosis (SWRO) and membrane bioreactor (MBR) processes. ${ }^{7,8}$ And, due to their ability to produce high-purity water using simple-to-operate, modular designs with small footprints and with relatively low energy demands, membrane separations have continued to attract significant attention in the water treatment arena. ${ }^{9-11}$

Membrane separation processes used for the treatment of unconventional water supplies can be broadly categorized as desalination processes or hybrid membrane processes (Fig. 1). In the context of this review, membrane processes with the single purpose of producing potable water from a saline or wastewater source, even though they may require several stages (e.g., intake, pretreatment, and desalination), are classified as desalination processes. On the other hand, membrane processes with several integrated purposes such as harvesting energy, recovering resources, and generating non-potable water treated to fit the purpose of target applications are classified as hybrid processes. Desalination processes rely on membranes that are capable of permeating water while retaining all other dissolved solutes indiscriminately. Therefore, this approach, which forms the basis of SWRO, produces a permeate stream of potable water while a concentrated mixture of solutes is retained in the concentrate stream. While the approach has been successful at the large scale, it still faces fundamental challenges that need to be addressed before its adoption becomes more widespread. In particular, the management of the concentrate stream is one critical concern. In the case of SWRO, the concentrated brine can be disposed using appropriately designed approaches that reintroduce the brine into the ocean with managed environmental impact. ${ }^{12,13}$ The disposal of this brine, however, is a challenge for the desalination of inland sources (e.g., brackish water). Moreover, the large volume of brine has driven interest in zero liquid discharge operations that minimize the volume of the concentrate stream and maximize water production. ${ }^{13}$ However, the brine still contains micropollutants and other contaminants that pose risks to the environment and human health. Furthermore, state-of-the-art desalination membranes face materials challenges. For example, while the membranes are highly-selective, they still allow small amounts of solutes to permeate. ${ }^{14}$ This hampers the use of desalinated water

\footnotetext{
${ }^{1}$ Department of Chemical and Biomolecular Engineering, University of Notre Dame, Notre Dame, IN 46556, USA; ${ }^{2}$ Charles D. Davidson School of Chemical Engineering, Purdue University, West Lafayette, IN 47907, USA and ${ }^{3}$ Department of Chemistry, Purdue University, West Lafayette, IN 47907, USA

Correspondence: William A. Phillip (wphillip@nd.edu)
}

Received: 17 August 2017 Revised: 25 November 2017 Accepted: 7 December 2017

Published online: 26 April 2018 


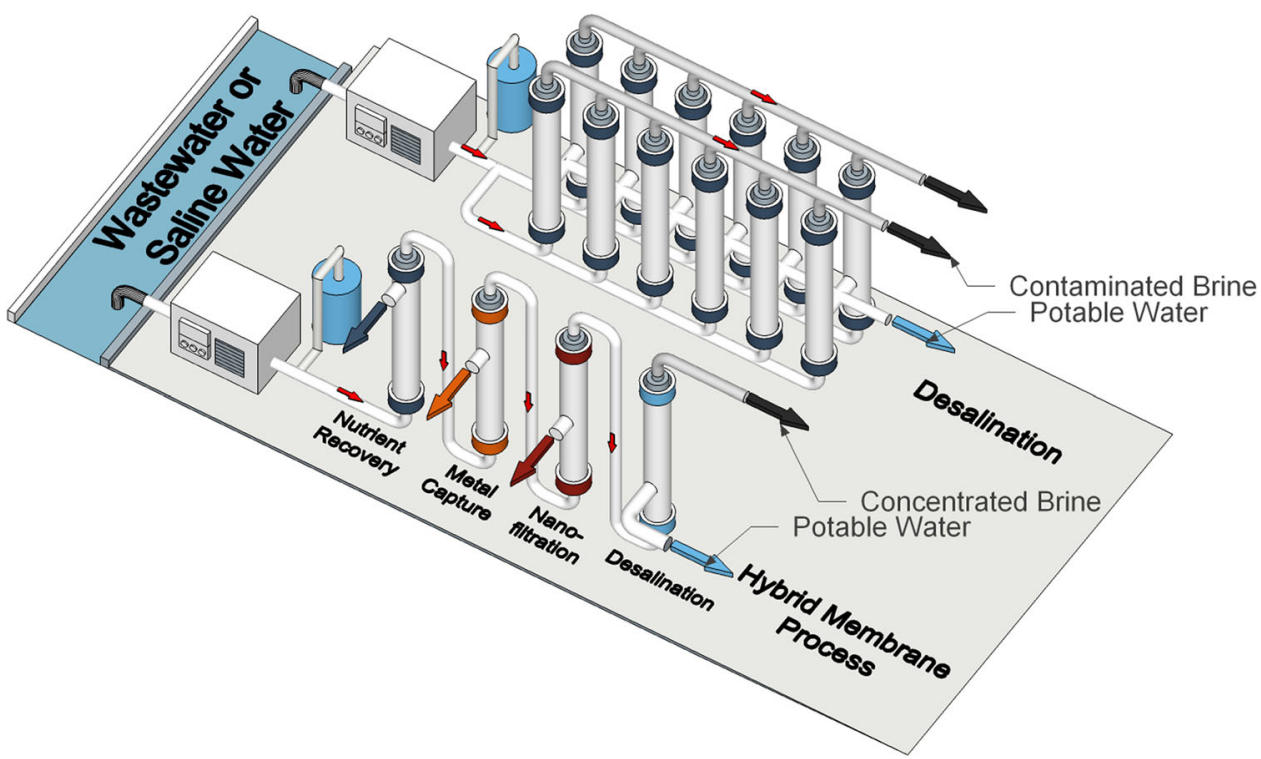

Fig. 1 A conceptual process flow diagram for water treatment by a desalination process and a hybrid membrane process. In contrast to desalination, which focuses on producing potable water as the end product, the hybrid membrane process is designed with a cascade of separations that target the generation of product water at the purity required for the end applications as well as the recovery of valuable resources. In this type of process, resource recovery and selective solute removal can be achieved by membranes with functional pore wall chemistries, as represented by each module, that are integrated in series. Many material platforms are in development for deployment in these processes. Self-assembled block polymer membranes have great potential for implementation in desalination and hybrid membrane processes due to their well-defined nanostructure and nearly-limitless surface chemistries

for agricultural purposes because the passage of boric acid and chloride ions through the membranes results in permeate streams that necessitate further processing to produce water that does not compromise plant health or quality. Finally, the susceptibility of the polyamide chemistry that constitute state-of-the-art desalination membranes to fouling and degradation by chlorine are other shortcomings in current designs. ${ }^{15}$ These issues highlight opportunities for the improvement of state-of-the-art desalination processes. Moreover, due to the thermodynamics of chemical separations, desalination processes will remain energy-intensive, which has driven interest in other sources of water.

Desalination processes are energy-intensive because they target the treatment of saline water sources, and the high osmotic pressures generated by these sources require large amounts of energy to overcome. This has caused engineers and scientists to consider the treatment of less saline sources of water (e.g., municipal and industrial wastewater) using novel processes, including membrane separations. While desalination membranes can be used to produce potable water from these sources, negative public perception resulting from the source water chemistry and incomplete removal of trace contaminants is a hindrance to direct potable reuse. Regardless, there are significant opportunities for non-potable water reuse to help meet the growing demand for this valuable resource, yet only a small fraction of wastewater is reused due to economic and technological constraints. ${ }^{16,17}$ There is a growing trend to view the nutrients, natural polymeric substances, and metal ions dissolved in wastewater streams as renewable resources that can be recovered. ${ }^{16,18-22}$ Such that, if the appropriate technological solutions can be developed, recouping the value of these resources may help ameliorate the economic costs associated with reuse. These factors have, therefore, driven research into the development of hybrid membrane processes that seek to treat water to the purity levels demanded by the requirements of its end-users, while also recovering useful resources from the feed stream. A preliminary example of this approach is in the use of nanofiltration (NF) membranes to remove divalent cations from saline water sources. The NF membranes do not, however, reject monovalent ions well, although the osmotic pressures (and associated energy demands) are less than the corresponding reverse osmosis process. While the level of water purity is not equivalent to that produced using reverse osmosis membranes, the permeate water still can be used or reused in several applications (e.g., cooling towers) without the worry of scale buildup due to the fact that the divalent ions have been removed. ${ }^{23}$ This is a single, but important, example of how novel separation technologies will be essential to the development of more sophisticated hybrid membrane processes that recover a variety of valuable solutes while producing potable or nonpotable water for target applications.

Hybrid processes, which utilize a cascade of separation processes to produce water of a desired quality while recovering useful resources from the feed stream, are envisioned in future reuse systems. ${ }^{24,25}$ Multifunctional membranes with deliberatelyassigned pore wall chemistries provide a platform for hybrid processes by moving beyond the conventional size-selective membrane separations that have dominated the field to date. These chemistries can be thoughtfully designed to effect selective separations through affinity-based adsorption, electrostatic interactions, and catalytic conversion of target solutes. In this manner, the separation is tailored based on the chemical composition of the source water with the end-use requirements in mind. Thus, targeted purification and solute capture can be accomplished through the integration of functionalized membranes with existing and other emerging water treatment technologies.

New membrane materials that address the limitations of stateof-the-art membranes or introduce new functionality will aid in the advancement of both system designs. This review focuses on one promising materials platform, namely, self-assembled block polymers and how their self-assembled nanostructure and nearly-limitless chemical compositions can be used to develop membranes, using scalable nanomanufacturing processes, for water treatment systems. First, a discussion of how block polymers are tailored at the macromolecular level for targeted 
membrane properties provides a foundation for the subsequent discussion. Next, the ability to rationally manipulate the nanostructure of block polymer membranes provides a basis for understanding how the limits of size-selective, self-assembled membranes are being pushed into the NF regime as well as the potential for the development of more selective desalination membranes from this materials platform. Subsequently, the ability to tailor the surface chemistry of block polymer membranes for different water reuse and resource recovery applications through a variety of modification mechanisms is discussed. With these large number of parameters to be considered, the phase space for experimental scientists and engineers to investigate could be limitless. Thus, the role of computational screening using molecular dynamics (MD) simulations for the evaluation of candidate chemistries tailored for targeted separations is addressed. The results that are calculated from simulation provide potential solutions in order to begin to establish the key structure-property-performance relationship of a membrane. In this manner, block polymer membranes with specific separation profiles can be designed and developed through a library of rationalized techniques to meet the spatial and temporal variations in composition among different sources of water intake and user demands. Subsequently, the integration of functional block polymer membrane operations could find a role in the development of processes that produce high quality clean water, recover valuable resources, and discharge environmentally responsible waste streams in a manner that addresses global water needs in an energy- and cost-efficient manner.

\section{TUNING THE NANOSTRUCTURE FOR SELF-ASSEMBLED MEMBRANES}

Equilibrium nanostructures demonstrate the potential of block polymer films as membranes

The self-assembled nanostructure of block polymers in the equilibrium state was what first suggested their utility in the development of high-performance membrane separation devices. Specifically, the high-density arrays of well-defined nanopores that developed were appealing for incorporation into a membrane, as they should lead to structures with high hydraulic permeability and separation selectivity (Fig. 2). ${ }^{26,27}$ This combination of properties could allow for the development of block polymer membranes that exceed the upper bound developed for commercial ultrafiltration membranes made using non-solvent induced phase separation (NIPS) processes. ${ }^{28-30}$ Because the potential of these membranes is related directly to their nanostructure, the deliberate design of the macromolecular architecture of the precursor block polymers is essential to the development of block polymer membranes. ${ }^{31} \mathrm{~A}$ fundamental criterion in this design is that the material microphase separates such that it forms ordered structures at the nanoscale. In most instances, this microphase separation is driven by the chemical incompatibility between the covalently-connected constituent moieties of the block polymer, as parameterized by the Gibbs free energy of mixing. For the simplest case of A-B diblock copolymers, the extent of incompatibility is quantified using a single segregation strength, $\mathrm{XN}$, where $\mathrm{X}$ is the interaction parameter between the two blocks and $N$ is the overall number of repeat units (after normalization to a given repeat unit volume). Based on the volume fraction $f_{\mathrm{i}}$ of each block, a critical value of $\mathrm{XN}$
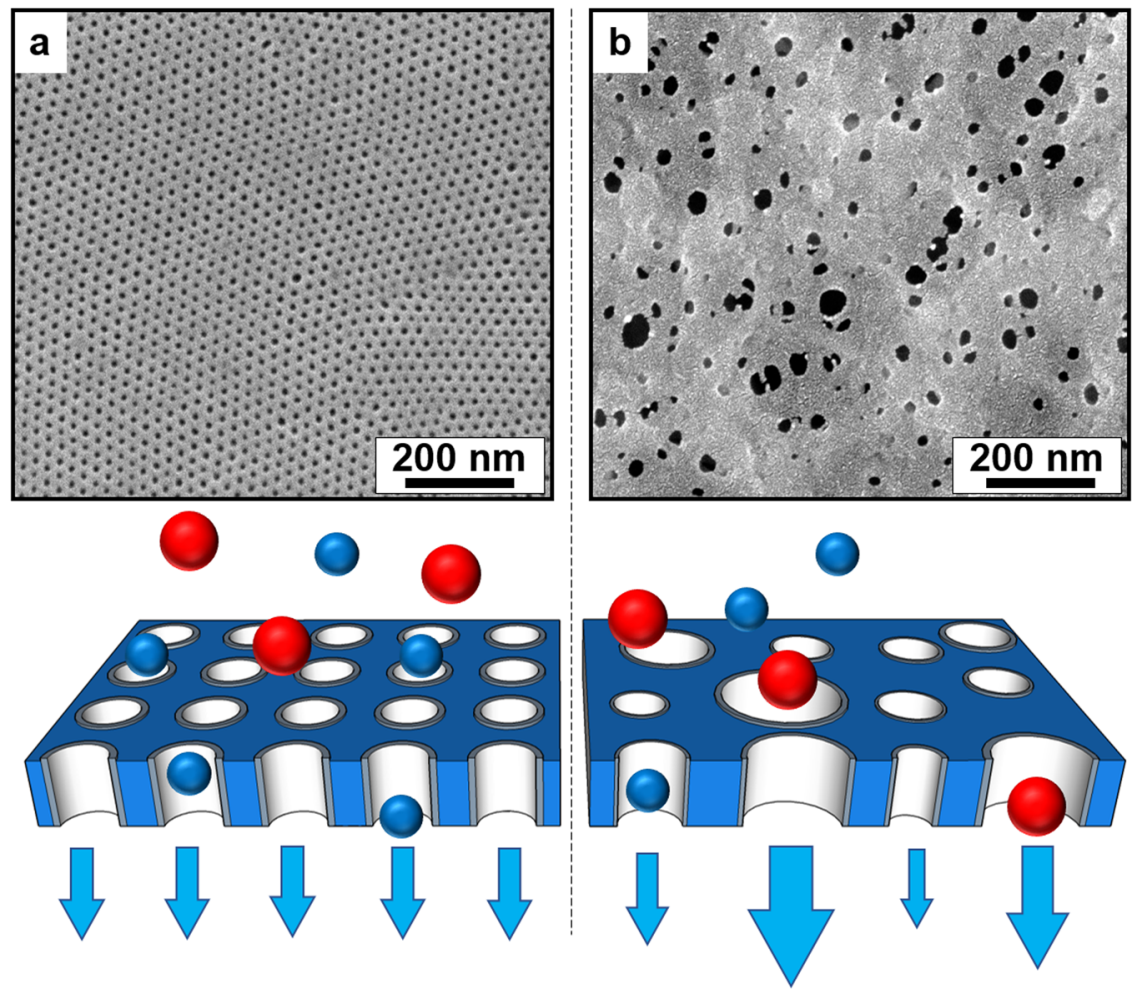

Fig. 2 Scanning electron micrographs of $\mathbf{a}$ a self-assembled block polymer membrane and $\mathbf{b}$ a commercial membrane made using a standard phase separation process. The highly-ordered nanostructure of the self-assembled membrane offers the potential for higher performance (i.e., higher throughput and more selective) separations relative to commercial membranes because the well-defined, narrow pore size distribution produces highly-selective filters, and generates a uniform flow distribution through each pore. The blue and red spheres represent solutes of varied size being filtered from solution. The width of the blue arrows is proportional to the volumetric flow through the associated pore. Solute selectivity and a uniform residence time for fluid flowing through the membrane pores are essential in the development of functional membranes for advanced applications. a adapted with permission from ref. ${ }^{35}$, copyright 2010 American Chemical Society 
must be exceeded for phase separation to occur. ${ }^{31-33}$ Above the minimum value of $X N$ in the phase diagram for $A-B$ diblock copolymers, the block polymer self-assembles into a variety of nanostrucutres, while below the critical value a disordered region is observed. Experimental and theoretical studies, which are discussed elsewhere, have identified the self-assembled nanostructures that develop for diblock and multi-block polymers as a function of composition. ${ }^{31,33}$ Therefore, targeting an equilibrium nanostructure as a precursor for a membrane requires considering the block-block interactions, overall degree of polymerization, and the volume fraction for each moiety.

The synthesis of block polymers that self-assemble into the desired precursor morphologies in a reproducible manner is an indispensable step towards implementing these materials into membrane units with fit-for-use purification profiles. The large number of morphologies available through appropriate macromolecular design provides several nanostructures that could be implemented. This even includes the possibility of stacking the morphologies in successive layers. ${ }^{34}$ However, the need to ensure the concurrent formation of a permeable domain that percolates across the entire membrane thickness (without channels that are dead ends) and a mechanically-stable matrix provides a further constraint to the design of the materials. Based on these criteria, block polymers that self-assembled into bicontinuous or cylindrical morphologies have attracted broad interest. The initial studies into these materials as separations media focused on the transport characteristics of nanoporous block polymers. ${ }^{35-37}$ Therefore, these efforts implemented processing routes that resulted in the development of porous and permeable materials not necessarily the formation of membranes using scalable processing methods. For example, thin films of block polymers can be prepared by coating a solution onto a solid substrate followed by complete solvent evaporation and annealing to an equilibrium nanostructure. In order to induce porosity, the thin film may undergo selective swelling in the melt followed by extraction of the swelling agent ${ }^{38-40}$ or a sacrificial moiety may be etched to create the percolating network of pores necessary for filtration. ${ }^{35,41-43}$ The nanostructured thin film is then transferred onto a mechanically stable support for transport characterization.

Using this approach, block polymer membranes with cylindrical domains that have their long axes orientated normal to the plane of the substrate have been studied in detail. The need to orient the cylindrical domains with their long axes perpendicular to the membrane surface while ensuring that they span the entire membrane thickness is one design challenge to their use. Cylinders with a parallel orientation or pores that dead-end before traversing the membrane result in impermeable domains. Unfortunately, both of these structural features are often found in practice. $^{36,44-47}$ However, carefully controlling processing conditions through: (1) regulating the rate of solvent evaporation; (2) tailoring the substrate block polymer interactions; (3) and/or selective solvent annealing have allowed for this challenge to be met, and supported thin films with cylindrical pores have demonstrated excellent performance in filtration applications. $^{35,41,42,43,48,49}$ For example, membranes based on polystyrene- $b$-poly (methyl methacrylate) (PS-PMMA) were used to filter a virus from solution. ${ }^{43}$ After coating the block polymer on a functionalized substrate, the pore-forming PMMA moieties were etched using a combination of exposure to ultraviolet light and acetic acid. The resulting nanoporous PS thin film was then transferred to a commercial support for filtration studies. When compared with commercially-available standards, the nanoporous PS thin film demonstrated higher fluxes and selectivity. Since this seminal study, several other efforts have used this approach to generate key insights into the use of nanoporous block polymer thin films based on cylindrical morphology as filters. ${ }^{3,40,50-55}$

\section{Scalable fabrication of high performance block polymer membranes}

The self-assembled nanostructure of block polymers at equilibrium demonstrated their promise as next-generation membranes. However, the translation of these materials to functional membrane systems required more than a well-ordered nanostructure on the surface. The high-throughput fabrication of an asymmetric cross-section that consists of a thin (i.e., $100-500 \mathrm{~nm}$ thick) self-assembled active layer supported by an underlying microporous layer is needed for the promise of these materials to be realized. In this regard, the self-assembly and non-solvent induced phase separation (SNIPS) technique, which combines selfassembly of the block polymers in solution and controlled solvent evaporation followed by the membrane industry standard NIPS process, has received the most attention because it can lead to the formation of an asymmetric structure without post-fabrication treatments to create the permeable domains (Fig. 3). ${ }^{56,57}$ The SNIPS process begins by casting a thin film of a block polymer solution on a substrate followed by a period of controlled solvent evaporation. The evaporation of solvent generates an increased polymer concentration at the solution-air interface, which causes the block polymer to form into ordered nanostructures across the top surface of the film. ${ }^{56,58-61}$ At this juncture, it is critical to note that these nanostructures are dynamic and driving toward equilibrium such that the instantaneous nanostructure observed at any point during the evaporation step depends upon the solution composition, solvent evaporation rate, and block polymer properties. At the end of the solvent evaporation step, the film is plunged into a non-solvent bath. By plunging the film into the
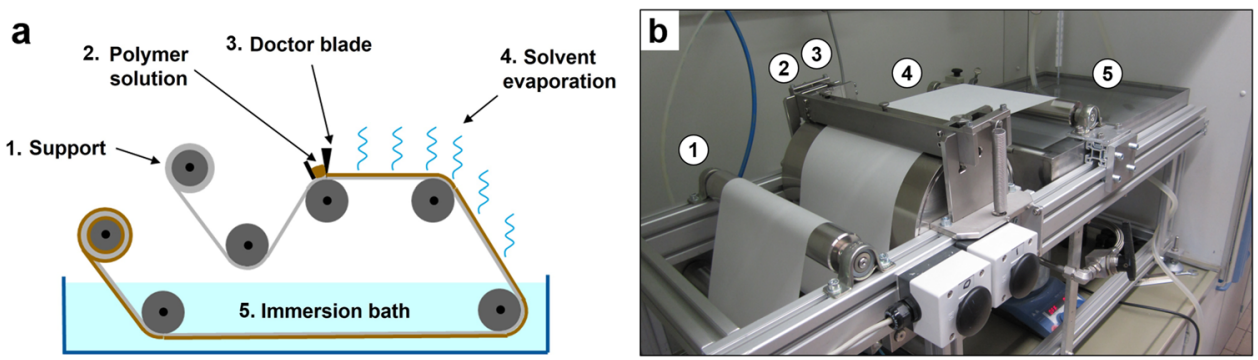

Fig. 3 a A schematic representation of the SNIPS membrane fabrication protocol. In the SNIPS process, a self-assembled block polymer is dissolved in a solvent to form a homogenous solution that is drawn into a thin film on top of a porous support (e.g., a nonwoven fabric) using a coating blade. The solvent is then allowed to evaporate for a pre-determined amount of time prior to plunging the thin film into a nonsolvent bath. During the solvent evaporation period, the polymer concentration at the solution-air interface increases, which drives the formation of a self-assembled nanostructure in the active layer of the membrane. Introducing the film into the non-solvent bath, causes the polymer to precipitate, which traps the self-assembled nanostructure of the active layer and causes the phase inversion of the support layer. $\mathbf{b}$ A photograph of a laboratory-scale roll-to-roll casting machine used to implement the SNIPS membrane fabrication process. $\mathbf{a}$ and $\mathbf{b}$ adapted from ref. ${ }^{57}$, copyright 2014 Elsevier 
non-solvent bath, the rapid exchange of solvent and non-solvent precipitates the block polymer and traps the self-assembled nanostructure of the active layer. This exchange also drives the formation of the microporous structure in the underlying support. Because the lower portion of the thin film is far from the solution interface, the evaporation front does not have time to travel across the film thickness. Therefore, the morphology in the bottom portion of the film is determined by the instantaneous composition when the non-solvent bath is initiated and the rate of solvent-non-solvent exchange, as studied in detail for the NIPS process. The resulting support or gutter layer possesses larger pore sizes that, in the ideal case, do not contribute a significant hydraulic resistance to the asymmetric membrane. This summary provides a useful framework for considering the design of SNIPS processes. However, ample room for understanding the detailed structure formation mechanism at the nanoscale, especially for self-assembled morphologies far from the equilibrium morphologies predicted in the bulk material, exists.

To address these issues, studies aimed at identifying design rules for the SNIPS process based on fundamental scientific principles have begun to emerge. These studies began by examining the self-assembly of the block polymer in the casting solution, which experiments suggested was a critical parameter in determining the morphology of SNIPS fabricated membranes. In particular, the solvent identity and polymer concentration in the casting solution must be selected appropriately. Solvent selection is guided by two general principles with respect to the need to produce: (1) self-assembled, porous nanostructures and (2) an asymmetric membrane cross-section. The formation of these two structural motifs is directly related with the interaction between the solvent and the block polymer, the volatility of the casting solvent, and the rate of exchange between the solvent and nonsolvent. In regards to the first point, small angle X-ray scattering (SAXS) and cryogenic-transmission electron microscopy (cryoTEM) data have revealed the role that solvent selectivity plays in the nanostructures nucleated within the casting solution. Specifically, solvent selectivity may impact the effective segregation strength and volume fraction of the block polymer, leading to the formation of morphologies in the initial casting solution that are different than those observed in the bulk material. ${ }^{62,63}$ For example, block polymers that form cylinders in the bulk can produce micelles in solution that then propagate to various nanostructures in the active layer of the final membrane. ${ }^{59,63,64} \mathrm{~A}$ discussion regarding the effect of solvent selectivity in the formation of these nanostructures has been detailed in other reviews. ${ }^{29,65}$ Of particular note, a semi-empirical trend line, which was related to the block polymer composition and solvent interactions with each constituent block, was introduced to serve as a guide for the identification of selective solvents that drove micelle formation when new block polymer precursors were being considered for the fabrication of membranes. ${ }^{64}$ Also related to the second point that guides solvent selection, the vapor pressure of the solvent should be sufficiently high such that, upon evaporation, a gradient in the solvent concentration is generated across the film. This leads to an asymmetric cross-section with a selfassembled active layer situated on top of a support layer.

Time-resolved cryo-TEM, small angle neutron scattering (SANS), ${ }^{6-69}$ and SAXS data have recently been utilized to examine the dynamic evolution of the self-assembled nanostructure within the active layer during the solvent evaporation step of the SNIPS protocol (Fig. 4). As solvent evaporates, the surface nanostructure undergoes a transition from a disordered morphology in the semidilute regime into various ordered states as a function of the polymer concentration. . $^{6} 68,70,71$ For example, in a study with polyisoprene- $b$-polystyrene- $b$-poly(4-vinylpyridine) (PI-PS-P4VP) membranes, the block polymer in the cast film experienced a transformation from a body-centered cubic lattice into a simple cubic as the evaporation process progressed. This simple cubic lattice was evident in the terminal nanostructure of the membrane surface (Fig. 4c). As such, these studies are critical to understanding the transitions between morphologies that occur at different periods during the solvent evaporation process, and further inform the choice of the evaporation step length, solvent composition, and initial concentration of polymer in the casting solution. In addition to the effects of solvent evaporation time, several studies have demonstrated that the terminal selfassembled nanostructure of the membrane is sensitive to environmental factors such as humidity or the temperature of coagulation bath during the casting process. ${ }^{72,73}$ Thus, the appropriate selection of these parameters, which are controlled in large-scale membrane manufacturing processes, is critical to the reproducibility of SNIPS derived nanostructures.

The solvent selected for the SNIPS process should produce asymmetric membranes with cross-sectional structures that are designed to realize the full potential of the block polymer active layer. In other words, the underlying support layer cannot provide a significant resistance to flow (Fig. 5). The architecture of the underlying support or gutter layer is determined by the precipitation pathway through the ternary phase diagram for polymer-non-solvent-solvent mixtures. ${ }^{74}$ Therefore, the morphology of this layer depends on the local composition of the solution and the rate of solvent exchange at the instant the non-solvent precipitation bath is initiated. Because it is the industrial standard for membrane fabrication, an extensive literature exists regarding the NIPS process. ${ }^{74}$ These prior studies demonstrate that, for a given polymer, the choice of solvent and non-solvent can be adjusted to produce highly-porous cross-sectional structures. However, the constraint that the block polymer should selfassemble within the active layer restricts the modifications that can be made in this regard. For this reason, strategies for tailoring the cross-section have been focused on utilizing additives and facile modifications to the membrane fabrication process. Strategies to control the gutter layer structure have been studied extensively for the PI-PS-P4VP system with the chemical composition and temperature of non-solvent bath and the introduction of additives in the casting solution being examined systematically. ${ }^{72,73}$ For example, the inclusion of additives that modify the rate of solvent exchange (i.e., $\mathrm{TiO}_{2}{ }^{75}$ and glycerol ${ }^{76}$ ) in the casting solution drove the formation of a highly-porous support layer. In these cases, the cross-sectional structures do not contribute significantly to the overall hydraulic resistance and the potential of the self-assembled block polymer membranes has been realized.

In addition to tailoring the cross-sectional morphology by modifying the phase inversion step of the process, researchers have developed thin film composite membranes by using novel solution deposition protocols, in lieu of blade coating, at the outset of the process. These technologies highlight the possibility to use less of the block polymer material by coating ultra-thin layers on a support in a controlled manner. For instance, spray technologies were used to coat $1 \mu \mathrm{m}$-thick films of polystyrene- $b$ poly(4-vinylpyridine) (PS-P4VP) on a support. These composite films exhibited a higher permeability than a $30 \mu \mathrm{m}$-thick membrane prepared from the same material by blade casting. ${ }^{77}$ Dip-coating offers another facile deposition procedure that provides systematic control over the total membrane thickness. ${ }^{78}$ Using this approach, the cross-sectional architecture has been controlled by modifying the dip-coating speed and evaporation time. At a low coating speed and longer evaporation times, a 500 $n$ m-thick poly(isoprene- $b$-styrene- $b$ - $N, N$-dimethylacrylamide) (PIPS-PDMA) layer was deposited atop of a hollow fiber support (Fig. 5c). These processing techniques give rise to additional adjustable parameters that enable further control of the crosssectional morphology and performance of the membrane. Regardless of the end application, the knowledge being generated by the studies discussed above is essential for the 

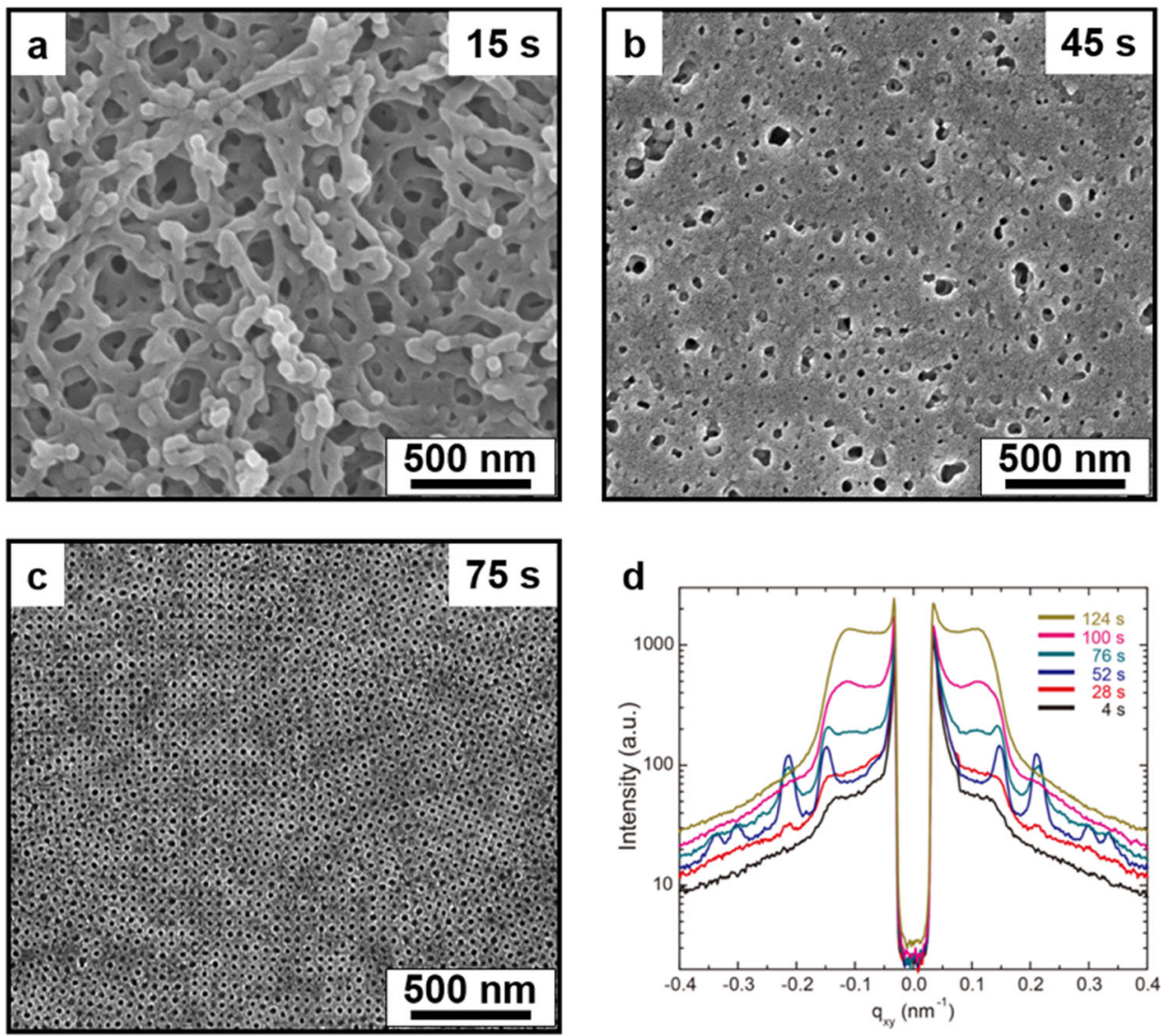

Fig. 4 The SNIPS technique is a dynamic process where the nanostructure of the membrane surface evolves as the evaporation period progresses in time. At the end of the evaporation period, the polymer solution is plunged into a non-solvent bath to vitrify the nanostructure on the membrane surface. a-c The kinetically-trapped surface nanostructure of PI-PS-P4VP membranes precipitated after evaporation periods of varying lengths of time. a Short evaporation times lead to a morphology similar to that observed with simple non-solvent induced phase separation casting processes. b Prolonged evaporation times initiated nucleation of a self-assembled naostructure. c At a sufficient evaporation time, the self-assembled nanostructure, with pores organized on a simple cubic lattice, covers the membrane surface. $\mathbf{d}$ In situ materials characterization techniques are invaluable tools in further understanding and designing the SNIPS process. For example, in situ GISAXS data demonstrate the structural evolution of a PI-PS-P4VP material during solvent evaporation. In this figure, the intensity of the scattering is plotted as a function of the magnitude of the scattering vector, $q_{x y}$. The material exhibits a transition from a disordered to an ordered morphology as the evaporation time increases from 4 to $52 \mathrm{~s}$ as indicated by the appearance of the well-defined peaks at higher values of $q_{x y}$ (i.e., higher order peaks), which is consistent with the onset of structure observed in SEM analysis. a-c adapted with permission from ref. ${ }^{58}$, copyright 2011 American Chemical Society. d Adapted with permission from ref. ${ }^{70}$, copyright 2016 American Chemical Society
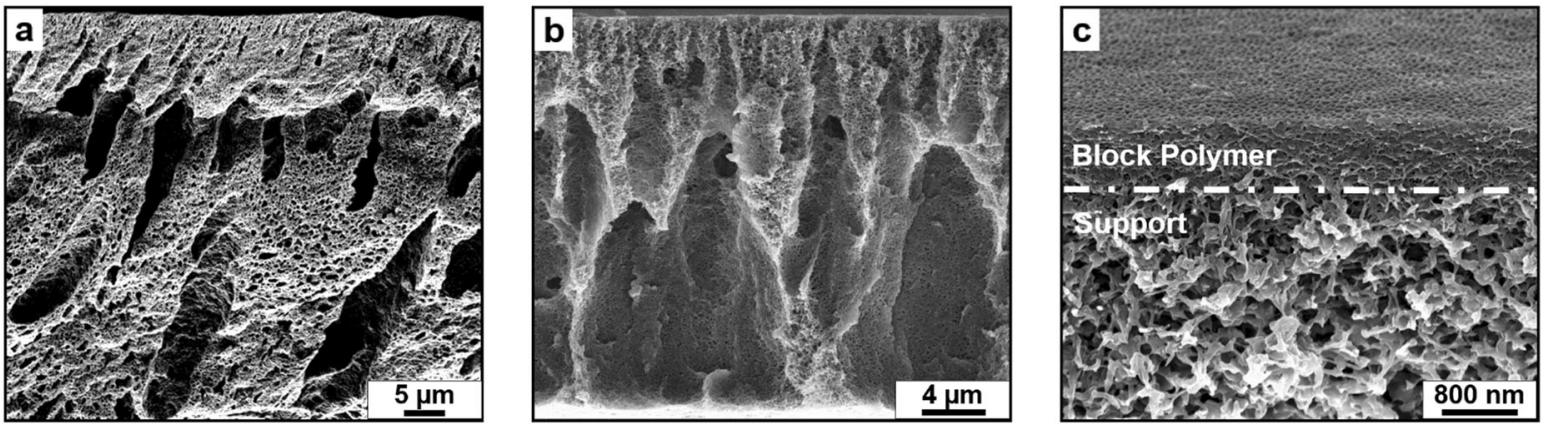

Fig. 5 The generation of high-performance membranes requires cross-sectional architectures that do not present a significant hydraulic resistance to flow. The cross-sectional morphology can be controlled during membrane fabrication by modifying the polymer deposition technique and the SNIPS process parameters. Three PI-PS-PDMA membranes demonstrate some of the cross-sectional morphologies that can be generated in this manner. a Sponge-like and $\mathbf{b}$ finger-like morphologies were generated by modifying the casting substrate. The highlyporous structure of the finger-like morphology resulted in a membrane with a significantly higher permeability. c An ultra-thin PI-PS-PDMA membrane fabricated atop a macroporous support by combining dip-coating and the SNIPS process. a adapted with permission from ref. ${ }^{92}$, copyright 2014 Elsevier. b adapted with permission from ref. ${ }^{78}$, copyright 2017 Royal Society of Chemistry. c adapted with permission from ref. ${ }^{93}$, copyright 2017 American Chemical Society 
rational design of block polymer precursors and the subsequent processing routes that transform them into high-performance membranes. Consequently, the well-defined nanostructure of selfassembled block polymer membranes and the molecular design versatility and flexibility associated with their chemistry enables the opportunity for their application in desalination and hybrid membrane processes.

Pushing the limits of self-assembled membranes: moving from ultrafiltration to desalination

The advances in identifying systematic approaches to tailoring the structure of block polymer-based membranes are enabling researchers to push the boundaries of where these materials can be deployed within water treatment processes. For sizeselective filtration membranes, a major research objective has recently centered about tuning the membrane pore size through molecular engineering. ${ }^{57,79}$ In this regard, fabricating membranes with smaller pore features has been identified as a critical direction of research for extending the applicable range of block polymer membrane devices into the nanofiltration and reverse osmosis regimes useful for desalination. To date, most block polymer membranes have been prepared with pore diameters in the range of 10 to $50 \mathrm{~nm}$. Yet, the pore size must be reduced to the $0.7-0.9 \mathrm{~nm}$ scale in order to be in the range where block polymers can be used for desalination. ${ }^{80-83}$

The feature size of microphase separated block polymers is related to the overall degree of polymerization. Therefore, one straightforward route to reducing the pore size of block polymer membranes, which has been implemented successfully in the ultrafiltration regime, is to use lower molecular weight precursors. However, this technique faces some challenges in order to achieve separations in the targeted desalination range. Specifically, as the overall degree of polymerization is reduced, the value of $x N$ can become too low for self-assembly to occur due to a lack of a thermodynamic driving force. ${ }^{84}$ The development of high $x$ block polymers, which exhibit large dissimilarities between each block, allow for the synthesis of low $N$ materials that self-assemble with smaller features. Due to their extremely hydrophobic nature, silicone-containing block polymers possess high $X$ values that make sub- $5 \mathrm{~nm}$ dimensions viable. ${ }^{85-88}$ For example, oriented cylinders with a $5 \mathrm{~nm}$ diameter could be generated from maltoheptaose- $b$-poly(trimethylsilylstyrene) materials. ${ }^{87}$ Furthermore, the use of charged moieties in one block induced additional charge cohesion that shifts the equilibrium phase diagram and led to self-assembly at low overall degrees of polymerization. ${ }^{89}$ Other examples of how polymer chemists are pushing the boundary of high $\mathrm{X}$-low $N$ materials are discussed in a recent review. ${ }^{90}$

A major concern during the design of such materials for membrane applications is the reduced mechanical integrity associated with the lower molecular weights, whereby the membranes become too fragile for practical applications. This is of particular importance in practice because large applied pressures are needed to overcome the osmotic pressures generated in desalination systems. The viscoelastic response of block polymer materials is an active area of research and progress in that arena provides a number of potential avenues for designing nanoporous materials that can withstand operation in high pressure membrane systems. Notably, while initial studies on block polymer membranes focused primarily on diblock materials, more recent efforts have incorporated a third rubbery moiety in the block polymer design to improve mechanical resilience. For example, the toughness of nanoporous membranes made from the triblock, polyisoprene- $b$-polystyrene- $b$-poly(4-vinylpyridine), was three times higher than the toughness of membranes made from the diblock analog, polystyrene- $b$-(4-vinylpyridine). ${ }^{58}$

Alternately, the careful design of the precursor material to include a reactive matrix that can be cross-linked may be a potential solution to preventing the collapse of nanostructured pores. In principle, because the distinguishing line between a low $N$ block polymer and a small molecule surfactant is not well defined, a successful approach to fabricate mechanically robust materials with a pore diameter below $2 \mathrm{~nm}$ has already been demonstrated through the directed assembly of liquid crystals with a magnetic field followed by crosslinking of the matrix. ${ }^{91}$ This system required the precise molecular design to include a magnetically anisotropic moiety that responded to the external field by aligning into highly ordered morphologies. However, it demonstrated the principle that mechanically-robust, selfassembled materials with small pore sizes are achievable.

An alternative approach to mechanically-robust block polymer membranes is the use of materials that can be post-synthetically modified to introduce chemical functionality that narrows the pore size into the desired range. For example, the implementation of polyelectrolyte brushes that are covalently attached within the nanopores of a block polymer membrane have been shown to swell upon hydration and lead to pores smaller than $5 \mathrm{~nm}$ in diameter from mechanically stable building blocks. ${ }^{76}$ A membrane with a pore diameter of $2 \mathrm{~nm}$ that exhibited sub-nanometer selectivity during filtration experiments was achieved through the use of pores lined by a high density of poly(2-acrylamido-ethane1,1-disulfonic acid) (PADSA) brushes. A PI-PS-PDMA material served as a precursor for these membranes. The pore walls of these membranes were initially lined by $\operatorname{poly}(\mathrm{N}, \mathrm{N}$-dimethylacrylamide) brushes, which were hydrolyzed to form poly(acrylic acid) $(\mathrm{PAA})^{78,92}$ that was subsequently converted to PADSA through a simple carbodiimide coupling mechanism. ${ }^{93}$ In such systems, which relied on the swelling of polymer brushes to narrow the pore size into the nanofiltration regime, the design of the brush chemistry was critical. Because weak polyelectrolyte (e.g., PAA or P4VP) brushes often respond to external stimuli through conformational changes that affect the pore geometry, a strong polyelectrolyte was preferred in order to generate a membrane with a consistent and small pore size. Block polymer membranes in the nanofiltration regime have also been realized through the blending of A-B/A-C type block polymers. In one recent study, the combination of PS-P4VP and polystyrene- $b$-poly(acrylic acid) (PS$P A A)$ led to small pore sizes due to the formation of hydrogen bonds between the PAA and P4VP blocks of the two materials. These complexes induced a morphological shift to densely-packed spherical nanostructures that resulted in small pore sizes. ${ }^{94}$ These efforts demonstrated that the selectivity of block polymer membranes is retained while shifting the pore size from the ultrafiltration regime into the nanofiltration regime. The successful demonstration of these nanofiltration test beds leaves the development of self-assembled membranes in the RO regime as the next critical separation regime to enter, which could address the material challenges of state-of-art systems in pursuing more selective desalination membranes. While no prior study has demonstrated such a membrane from block polymer precursors, computational studies, in particular molecular dynamics (MD) simulations, have provided critical information to guide the design of membranes targeted for this application by providing molecular-level insights into the selection of an ideal nanostructures for ion rejection.

By being able to track in detail the dynamic trajectories of (representative) water molecules and ions around and within model membranes, MD simulations have identified the salient interactions that are relevant to solute rejection. To first order, solute rejection is determined by the effective pore size of the membrane. When simulating membranes for desalination, an optimized pore size is selected based on the size of the hydrated solute. In the case of salt ions, a hydration shell of water molecules surrounds the ion. Hence, the entry of ions into small pores is made more difficult because the solvation shell first has to be removed in order for the ion to enter the pore. For instance, the 
diameters of hydrated sodium cations and chloride anions are 6.4 $\AA$ and $7.7 \AA$, respectively, while the thickness of the water shell surrounding an ion is between $2.5 \AA$ and $3.3 \AA .{ }^{95}$ Therefore, a starting point to selecting a pore size for salt rejection would be to choose a diameter that is smaller than the hydrated ion. However, because the sizes of the water molecules and ions are relatively close, and each one may interact with the membrane surface and pore walls differently, the observed trends of the effective salt rejection are not solely predicted by the sizes of the hydration shells of the ions. In fact, a more precise understanding of the salt rejection may be obtained by determining the potential of mean force (PMF) of ions along the pore from an MD simulation. In general, the PMF of a solute yields the free energy differences that arise upon changes in location of the solute, and therefore, provides information about molecular-level rearrangements that must occur (e.g., changes in solvent structure) upon translation of the solute. Here, the PMF provides a measure of the free energy barrier for the entry of ions into a membrane, and as such, yields important insights into the various molecular and structural rearrangements (including both those of the water within the hydration shell surrounding an ion and the polymers within the membrane) that occur as the ion enters a pore.

Previously, the PMF obtained using MD simulations of carbon nanotubes of different pore diameters ranging from $0.66 \mathrm{~nm}$ to $1.09 \mathrm{~nm}$ demonstrated that there is a strong correlation between the free energetic barrier for ion entry and the size of the pore. ${ }^{96}$ For a $0.66 \mathrm{~nm}$ diameter pore, the free energy change upon entry of $\mathrm{Na}^{+}$was found to be $23 \mathrm{kcal} \mathrm{mol}^{-1}$, while the free energy barrier was only $3 \mathrm{kcal} \mathrm{mol}^{-1}$ for a $0.93 \mathrm{~nm}$ pore diameter. Hence, for small enough pores, the membrane provides a high resistance to the entrance of the water shells that surround the ions, and yields the expected high resistance to the ions themselves. But that resistance rapidly decreases with an increase in the pore diameter. As the probability of an ion entering the pore is inversely proportional to the exponential of the free energy barrier, the $\sim 50 \%$ increase in the pore diameter led to a significantly greater chance of the ion entering the pore as it resulted in a nearly $90 \%$ reduction in the free energy barrier. For larger diameter nanotubes, fewer water molecules within the hydration shells need to be removed for the ions to enter the pore; a smaller dehydration barrier therefore makes ion permeation much more likely. On the other hand, those "unbounded" water molecules not within the hydration shell of a given ion were found to have a much lower free energy barrier for these same two pore diameters, $0.8 \mathrm{kcal} \mathrm{mol}^{-1}$ and $0.3 \mathrm{kcal} \mathrm{mol}^{-1}$, respectively. ${ }^{97}$ Therefore, these MD simulations demonstrate that the key mechanism of ion rejection in these systems is not simply the pore diameter but is instead the free energy cost associated with dehydrating the ion such that it can enter the carbon nanotube pore. Moreover, this study suggests that MD simulations also can be used to identify those changes to both the pore structure and chemistry that are needed to yield an appropriate free energy profile for optimal material transport. While primarily examined for carbon nanotube-based separations to date, these same types of MD simulations can, and should, be employed as a complementary tool for the rational design of block polymer membranes with targeted properties.

\section{MODIFYING THE PORE WALL CHEMISTRY FOR ADVANCED SOLUTE SEPARATIONS}

Design of pore chemistry enables applications beyond filtration

Pushing block polymer membranes from the NF regime into the $\mathrm{RO}$ regime has the potential to meet the emerging demand for more selective desalination membranes that allow for the passage of water while completely removing all dissolved solutes. This development alone, however, will likely not be sufficient to meet the world's thirst for water in a sustainable manner due to the high energy use associated with desalination processes. ${ }^{98}$ This need for less energy-intensive methods of producing usable water has led to interest in potable and non-potable water reuse as well as resource recovery processes. In these cases, the indiscriminate removal of all solutes is often unnecessary or undesired. Instead, the water can be treated such that it is fit for its targeted use and the dissolved solutes of value are isolated. The well-defined nanostructure and array of chemistries associated with block polymer systems will allow for the development of designer separation devices tailored to the specific demands of these emerging processes. In particular, the ability to control the surface properties of block polymer membranes and produce devices that regulate solute transport based on membrane-solute interactions is crucial to their potential utility for the targeted separations envisioned in reuse and recovery processes.

Block polymer membrane systems that are chemically-modified for target applications are an emerging area of study. ${ }^{28,99}$ As detailed in some of the examples above, these chemical alterations can modify the physical properties of membranes fabricated from block polymers such that they display excellent size-selective separation abilities. In addition, the block polymer system can be modified using straightforward schemes for controlled properties that go beyond the size of the membrane pores. The incorporation of reactive entities along the pore walls has been used to convert a membrane into a reactor with a high density of active sites within a small volume. ${ }^{100,101}$ Alternatively, the addition of coordination chemistry allowed for the purification of feed solutions containing contaminants, such as heavy metal ions, by adsorption. ${ }^{102,103}$ These examples utilized the transverse direction of the pores to simulate a staged process as the solution passed through the membranes. In this configuration, the nanoscale dimensions result in short diffusion lengths from the centerline of the pore to the pore wall that reduce mass transfer limitations. Furthermore, the uniform pore sizes of block polymer membranes provide them with a unique advantage over most state-of-the-art membranes in these applications because the well-defined nanostructure results in a narrow distribution of residence times associated with each pore. ${ }^{104,105}$ This uniformlydistributed flow allows block polymer membranes to regulate mass transfer in a more efficient manner because it prevents channeling and utilizes the high packing density of functional groups confined within the pores more effectively. For example, in reactive membranes, a well-defined residence time allowed conversion to be controlled precisely through regulating the water flux. Membranes can also be used to enhance the transport of target solutes across the membrane using favorable enthalpic interactions between solutes and pore walls. This control over permeation of solutes lays the foundation for the development of membranes for water purification applications, including the removal of toxic contaminants or the concentration of valuable materials dissolved in water supplies.

Functional self-assembled membranes have been achieved in several ways (Fig. 6). These include: (1) incorporating the functional chemistries into the membrane precursor; (2) using the self-assembled membrane as a template and grafting molecules to the pore wall or back filling the pores with a functional material; and (3) designing a reactive moiety into the membrane precursor that allows for post-assembly introduction of functional chemistries. Importantly, each of these approaches has its advantages and drawbacks; thus, care must be taken when selecting the means of implementation. Ultimately, the methods will be judged by their ability to introduce a high density of active groups into a well-defined membrane structure in a safe and rapid manner that is consistent with commercial membrane manufacturing processes.

The desired chemistry can be incorporated directly into the precursor block polymer material during synthesis. This chemistry 

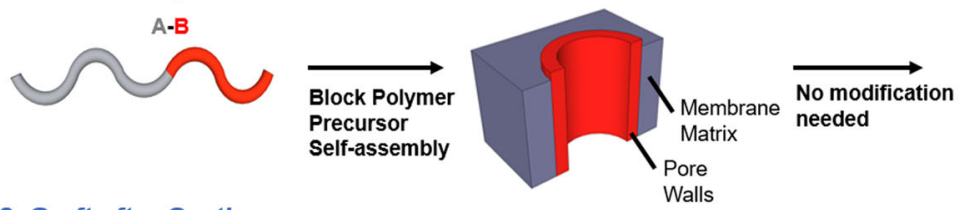

2. Graft after Casting
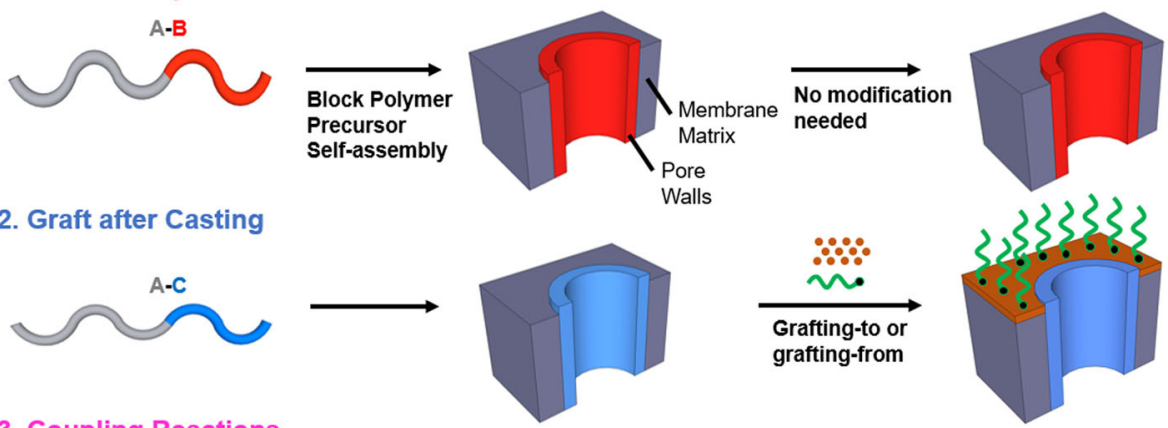

3. Coupling Reactions
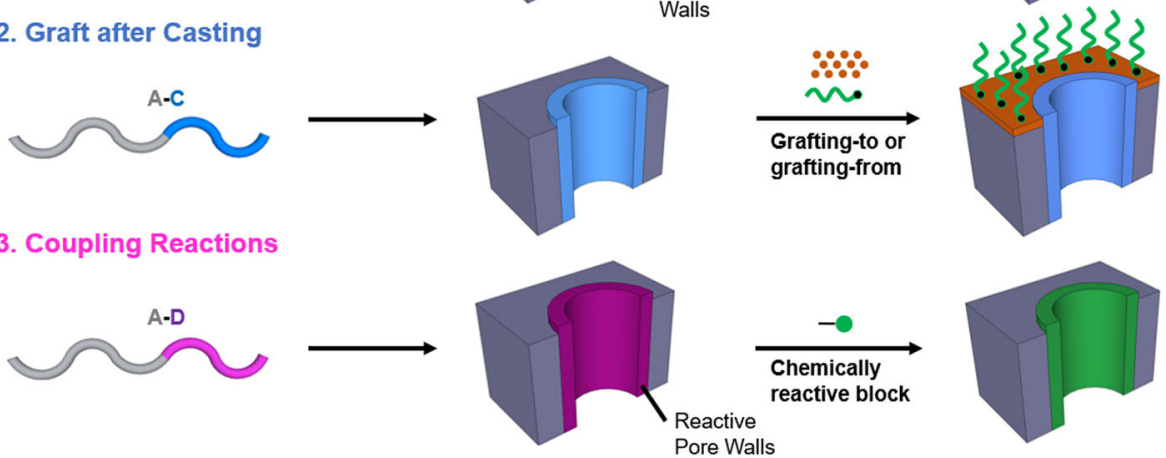

b

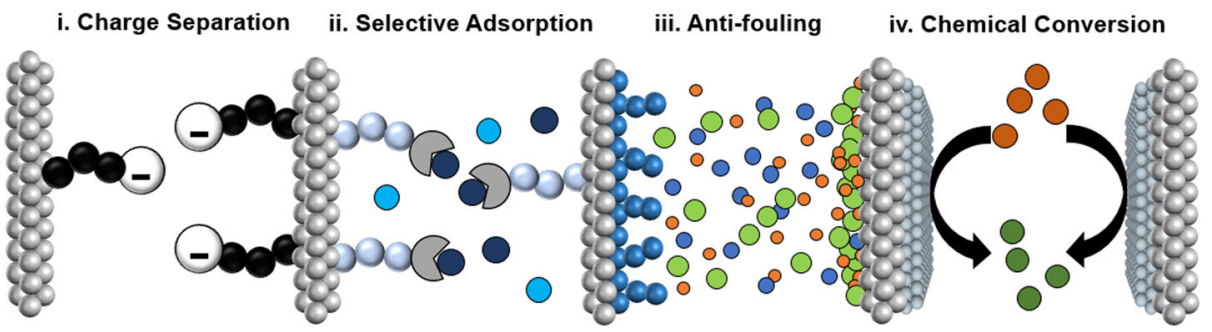

Fig. 6 a Functional moieties can be introduced into the pore wall chemistries of membranes made from block polymer precursors through three mechanisms. (1) The functional chemistry (red block) can be incorporated into the precursor block polymer prior to membrane fabrication. With the identification of suitable SNIPS parameters, a self-assembled membrane with functional pore walls that require no further functionalization reactions is generated. (2) The block polymer chemistry facilitates formation of a self-assembled structure. Subsequently, the surface of the membrane is modified using a coating (orange dots) to which functional chemistries are attached (green brushes). (3) The pore wall-lining block (magenta) is designed as a reactive chemistry that can be converted to a variety of functional chemistries through coupling reactions that are consistent with roll-to-roll processes. b An illustration of the broad range of potential applications where membranes with functional pore wall chemistries could be utilized

then becomes the pore wall lining moiety upon membrane fabrication, resulting in a membrane with a high "built-in" density of functional units. The P4VP block of PS-P4VP membranes is one example of this design approach. Whereby, the repeat units of P4VP were used to complex metal salts from aqueous solutions. $^{106-108}$ Subsequently, the salts that were localized within the membranes were reduced to form metallic nanoparticles that introduced catalytic functionality into the membrane. ${ }^{107}$ This study implemented a model reaction to demonstrate the catalytic activity, but the concept could be extended to the degradation of organic contaminants such that purification can be achieved through chemical conversion. Another iteration of this approach, which was discussed above, used the blending of chemically distinct block polymers (i.e., A-B and A-C types) to provide the desired functionality. ${ }^{109-112}$ While the minimal processing needed after membrane fabrication for this approach is attractive, the flexibility of the functional groups that can be used as the poreforming block may be slightly limited by the requirement that the material self-assemble during membrane fabrication.

Functionalizing the nanoporous structure with surface coatings following membrane fabrication is an alternative approach that circumvents the need to identify new SNIPS processing conditions for self-assembly each time a new surface chemistry is desired. The utilization of poly(dopamine) (PDA) chemistries is one example of a coating that has been used widely to attach functional groups without affecting the self-assembled nanostructure of the original membrane. ${ }^{113-115}$ The PDA coating enables modification using either the grafting-from or grafting-to approach. In one study, the polymerization of an antifouling 2hydroxyethyl methacrylate polymer brush onto the surface of nanostructured PS-P4VP membranes was achieved through the reaction of dopamine with 2-bromoisobutyryl bromide initiator, which then allowed the membrane to serve as an initiating site for polymerization using the atom transfer radical polymerization (ATRP) mechanism. ${ }^{114}$ Following a similar manner, the Michael addition reaction supports the grafting of functional molecules, such as zwitterionic L-cysteine or amine-terminated poly $(N$ isopropylacrylamide) (PNIPAM) to the dopamine without affecting the membrane performance. ${ }^{113,115}$ Additionally, the block polymer membranes can be used as sacrificial templates for the formation of nanoporous metal, metal oxide, and carbon films with welldefined nanostructures. ${ }^{116-121}$ The electronic and catalytic properties of these films could be useful in flow-through membrane reactors targeting the degradation of organic micropollutants and viral inactivation. This approach of adding desired chemical functionalities into a pre-formed membrane material allows for the incorporation of a range of chemistries with tunable properties. However, the need for a complex reaction scheme and long reaction times following membrane fabrication makes this approach a less attractive option for scale-up to roll-to-roll production.

Beginning with a polymer containing a block that, instead of incorporating the desired chemistry directly, can be chemically reacted to generate multi-functional devices after membrane fabrication enables another versatile approach to avoid the complexity of designing a new SNIPS procedure each time a 
new chemistry is desired. In this approach, rationally-designed reaction pathways can be used to modify surface properties through the covalent attachment of small molecules. The quaternization of the pyridine group in P4VP served as a preliminary example of this concept, as this chemical modification resulted in a positively-charged membrane surface. This made separations based on electrostatic interactions possible, as the positively-charged surface could separate solutes of similar size when one of the solutes was negatively-charged and the other was not. ${ }^{109,122}$ While further modifications were possible, the major shortcoming of P4VP is that a limited number of functionalization schemes are available. ${ }^{123}$

One example that tapped into a broader range of potential functional moieties for water purification is based on the previously described PI-PS-PDMA membrane, which contains pore walls that can be converted to PAA and subsequently to a variety of other chemistries. ${ }^{92}$ The membrane in the PAA-functionalized form was an effective adsorber for aqueous solutions containing copper, providing a high adsorption capacity and a sharp breakthrough once the binding sites were saturated. ${ }^{124}$ Because a variety of functional groups containing a primary amine can react with each repeat unit of the carboxylic acid lining the pore walls, these promising features were translated to applications more closely tied to water treatment processes. For example, a previous study demonstrated the coupling of the small molecule, cysteamine, as well as the peptide, glutathione, to the pore wall. ${ }^{125}$ Both chemistries were able to remove more than $98 \%$ of the lead ions in a $50 \mathrm{ppm}$ feed solution via selective binding between the metal ions and the thiol groups of the two ligands. Similar chemical flexibility can be realized in many precursor systems. Thiol-ene, copper(I)-catalyzed alkyne-azide cycloaddition, and other reaction mechanisms are attractive systems due to their modular nature as well as their rapid reaction kinetics. Recent studies have been focused on membranes equipped with a highdensity of functional groups amenable to several of these reaction mechanisms. ${ }^{126-131}$ The thiol-ene mechanism demonstrates the flexibility associated with these approaches. In one study, the covalent linkage is realized by using membranes that contain reactive thiol groups, such as polystyrene- $b$-poly(4-vinylpyridine)$b$-poly(propylene sulfide) (PS-P4VP-PPS) to attach the dye tetramethyl rhodamine- 5 to the membrane surface. ${ }^{128}$ Alternatively, the reaction mechanism is possible with solutes containing a thiol moiety through the incorporation of a reactive poly[(allyl glycidyl ether)-co-(ethylene oxide)] [P(AGE-co-EO)] domain within the membrane. ${ }^{126}$ Through the systematic macromolecular engineering of the block polymers, these coupling reactions should allow for a wide range of functional chemistries to be incorporated into membranes through the formation of resilient covalent bonds that form on a time scale consistent with the high throughput rollto-roll manufacturing of membranes on an industrial scale. ${ }^{132,133}$ This, in turn, will allow the vast number of functional membrane chemistries for targeted water-based separations developed within academic settings to be translated to larger scales.

\section{Modification of pore walls to charged groups for separation by electrostatic effects}

Charge-functionalized membranes highlight the utility of postsynthetically modified materials in the development of membranes for water treatment. For example, membranes with a larger pore size and, in turn, permeability that are functionalized with a charged moiety can achieve similar rejections as membranes with smaller pores due to repulsive electrostatic interactions between the dissolved ions and the pore walls. ${ }^{134,135}$ Using this paradigm, the development of membranes that selectively remove multivalent ions relative to monovalent ions, and that could be implemented in water softening and reuse applications, is possible through design of the surface chemistry. Moreover, the development of these membranes also highlights how experiments and MD simulations can be combined synergistically to guide their molecular engineering.

The selective rejection of divalent cations or anions was demonstrated experimentally through the introduction of ammonium and sulfonate groups, respectively. This trend in rejection was anticipated based on Donnan equilibrium, but the example demonstrated that the separation of ions can be tuned by simply controlling the functional groups lining the pore walls. ${ }^{135,136} \mathrm{~A}$ subsequent study, through a combination of MD simulations and experiments, revealed critical phenomena at molecular length scales that enhanced membrane design. The PMF for magnesium cations entering into a coarse-grained pore lined by positive charges demonstrated that charge-functionalized membranes effectively reduced the area available for ion permeation by increasing the free energy barrier for the entry of the co-ion (i.e., those with the same charge as the pore wall) into the pore. Moreover, the distance between the charged moiety and the pore wall (i.e., the spacer arm length) affected the free energy barrier for ion entry into the membrane. ${ }^{137} \mathrm{~A}$ consistent increase in the size of the free energy barrier was observed with increasing spacer arm lengths because the longer chains pushed the terminal charges toward the center of the pore and increased their repulsive coverage (Fig. 7b). Importantly, the molecular design versatility associated with the pore wall chemistry allowed for experimental validation of the simulation results (Fig. 7c).

Taken together, these studies highlight how chemical functionalization provides for additional separation mechanisms. They also demonstrate that designing the molecular architectures that provide the highest performance with respect to a targeted separations profile is challenging due to the myriad interactions that impact the affinity between the pore wall and the solutes of interest. Thus, a systematic approach to screening the pore structure and functionality is required, and molecular simulations can play an important, complementary role to meeting this need.

Tailored surface chemistries for solute-specific separations and controlled interfacial interactions

The benefits of adsorptive membranes based on block polymer materials are beginning to emerge. Notably, the high density of functional repeat units lining the pore walls result in large saturation capacities that are competitive with commercial resins. Therefore, the block polymer membrane platform has the potential to positively impact other challenging separations being studied in the field of water purification and resource recovery. For example, amidoxime binding chemistries for the targeted separation of uranium from seawater have been identified, but current substrates result in problematic low uptake capacities. ${ }^{138,139}$ The block polymer platform lends itself well to such instances due to its structural characteristics and easily modified chemical properties. These features could, likewise, improve the separation performance of membranes lined with phosphate-chelating agents for the recovery of phosphorus from wastewater streams. ${ }^{19,20}$ Moreover, the flexibility to freely modify these membranes with various chemistries could allow this platform to serve as a substrate for the incorporation of other highlyspecific binding moieties, such as cucurbiturils and cyclodextrins, which are effective agents for the capture of hydrophobic polarized molecules, respectively. In order to utilize the broad range of chemistries that can be introduced, and therefore separations that can be achieved, effective binding groups for solutes of interest must be identified. This search can be aided by the molecular-level detail that is provided by computational studies.

In MD simulations, the interactions of the functional group with the solute can be evaluated by measuring the coordination number and the binding affinity. The local solute atmosphere 

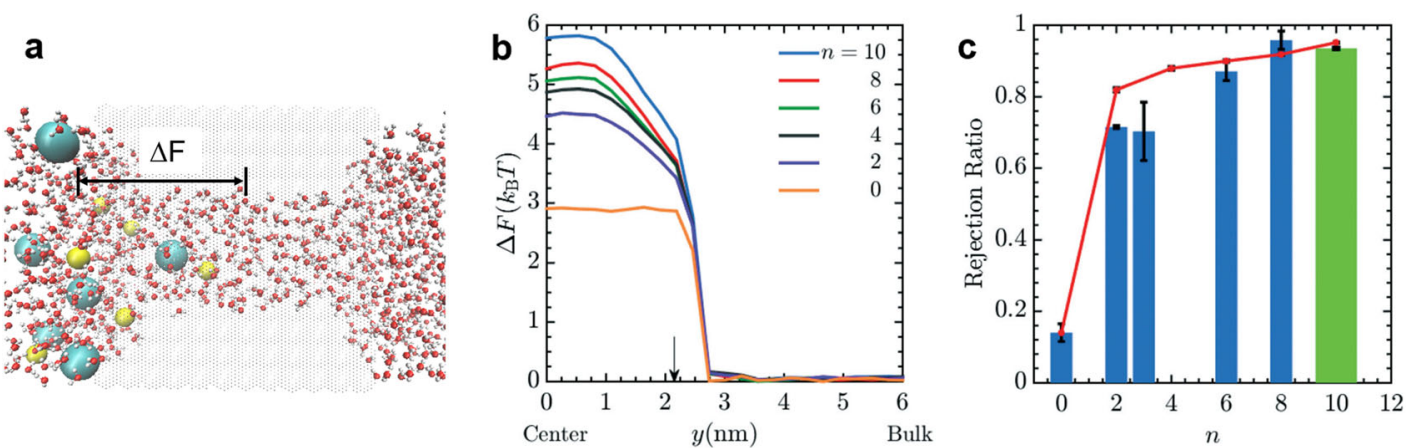

Fig. 7 Molecular scale information that guides the systematic design of pore wall chemistry can be determined through carefully constructed MD simulations. In this case, the rejection of magnesium ions through a positively charged membrane is used as a model. a Schematic showing the measurement of the free energy change of ions (blue and yellow) as they are entering the pore (atoms forming the membrane are gray). $\mathbf{b}$ The PMF profiles for the translation of a magnesium cation from a bulk solution into the pore of a membrane. The entrance of the functionalized pore is indicated by the arrow at $\sim 2 \mathrm{~nm}$. The orange line represents a parent membrane, $n=0$; the other lines correspond to simulations executed with increasing spacer arm lengths. The spacer arm length, $\mathrm{n}$, is defined as the number of carbon atoms between the pore wall linkage and the terminal charged functional group. c Simulated and experimental rejection for magnesium cations plotted as a function of spacer arm length, $n$. The simulation results, which were calculated using the free energy barrier obtained from $\mathbf{b}$, are shown with the red line. The experimental data, which are represented by the bar graphs, were measured using a $10 \mathrm{mM} \mathrm{MgCl}$ feed solution. $\mathbf{b}$ and $\mathbf{c}$ adapted with permission from ref. ${ }^{137}$, copyright Royal Society of Chemistry

around the functional group can be analyzed via the coordination number of the molecules within the first solvation shell of potential binding sites. Additionally, the binding affinity of different solutes to a functional moiety can be calculated with the PMF or, equivalently, the pair correlation function between the interaction sites and dissolved species, which here quantifies how the free energy changes as a function of the distance between the functional group and the solute. ${ }^{140}$ Understanding better the complex interactions between the solute particles and pore wall groups will allow for improved predictions of the affinity, and subsequently allow for the development of groups that present optimal binding affinity and specificity for the design of water treatment systems.

The ability to modify their surface chemistry rapidly can broaden the applications of this membrane platform to allow for their deployment in applications that require specific wetting properties or surface energies (e.g., membrane distillation). ${ }^{141,142}$ To date, due to its ubiquitous presence in water treatment processes, most membrane have been modified using surface chemistries aimed at mitigating the deleterious effects of fouling. Fouling, which is the adsorption of materials to membrane surfaces, is a limiting factor that affects the performance of almost all membrane separations through the loss of throughput. ${ }^{143}$ It has been clearly demonstrated that tuning the surface properties of the membranes can help to regulate the extent of fouling. ${ }^{144,145}$ And while general guidelines for the generation of surfaces with low fouling propensities have been established, ${ }^{146}$ they are not universally applicable and each water source may require specific surface chemistries. ${ }^{147}$ Therefore, the ability to make membranes with a well-defined nanostructure that are amenable to chemical modifications on time scales consistent with roll to toll processing presents an opportunity to implement surface chemistries that are designed based on the composition of the feed water.

\section{CONCLUSION AND OUTLOOK}

Desalination is an established separation process and will continue to be critical to the production of potable water from nontraditional sources. The large energy demand needed to execute the process, however, will necessitate the utilization of other unconventional sources to meet the global demand for water sustainably. As such, the design of multifunctional membranes that enable the design of hybrid processes for water reuse and resource recovery will be critical to tapping into non-traditional sources. The self-assembled block polymer membranes detailed in this review present several opportunities for the development of next-generation membranes that possess performance profiles that address the needs of desalination and hybrid membrane processes. In the past decade, the feasible pore size of block polymer membranes has been reduced an order of magnitude from the ultrafiltration regime $(\sim 20 \mathrm{~nm})$ to the nanofiltration regime $(\sim 2 \mathrm{~nm})$. In order to be utilized in reverse osmosis processes, the pore size will need to be reduced even further to $\sim 0.7-0.9 \mathrm{~nm}$. This will be a challenging transition, but the growing body of work that is currently elucidating how to generate structural precursors with the needed feature sizes and how to processes those precursors into membranes holds the promise that materials that are more resilient and more selective than current state-of-the-art desalination membranes can be developed. Furthermore, the modular design of block polymer materials allows for the ready incorporation of functional chemistries in a systematic manner. The membranes that result provide critical characteristics, such as a high density of functional moieties and uniformly distributed flow profiles, which enable the design of hybrid membrane processes capable of treating water to the levels demanded by end-user applications while recovering valuable resources. MD simulations provide a predictive tool to guide the design of these functional membranes such that chemistries that enable target separations with minimum energetic and chemical input are identified.

In lab-scale experimentation, block polymer membranes have demonstrated the potential to affect water treatment processes in a positive manner. Realizing the potential of this platform requires that the membranes are translated from the laboratory setting to practice. This transition necessitates collaboration between scientists and engineers to address questions that remain in regards to the large-scale fabrication of block polymer membranes as well as their long-term use in water treatment processes. Environmental conditions will need to be well-controlled in larger membrane fabrication processes to ensure alignment of the membrane nanostructure. In this regard, the development of inline quality control metrics would aid the reliable fabrication of self-assembled membranes. Furthermore, due to the variability of the feed streams that will be encountered, investigating the longterm pilot plant operation of block polymer membranes is essential for identifying the technological challenges associated with their use that cannot be simulated in laboratory settings. The ability to molecularly engineer self-assembled block polymer 
materials in order to overcome these new challenges will make these membranes an exciting and fruitful area of research and industrial application in the years to come.

\section{ACKNOWLEDGEMENTS}

Portions of this work were made possible with support from the Army Research Office (ARO) through the Polymer Chemistry Program (Award Number: W911NF-14-10229, Program Manager: Dr. Dawanne Poree) and the National Science Foundation (NSF) through the Interfacial Processes and Thermodynamics Program (Award Number: 1511835, Program Manager: Dr. Nora Savage) and through the Chemical and Biological Separations Program (Award Number: 1512089, Program Manager: Dr. Angela Lueking), and we appreciatively acknowledge this support. Y.Z. gratefully acknowledges support for this project from the CEST/Bayer Pre-Doctoral Research Fellowship at the University of Notre Dame.

\section{AUTHOR CONTRIBUTIONS}

Y.Z., N.A.A., J.L.W., D.S.C., B.W.B., and W.A.P. all contributed to the writing and editing of the review.

\section{ADDITIONAL INFORMATION}

Competing interests: The authors declare no competing interests.

Publisher's note: Springer Nature remains neutral with regard to jurisdictional claims in published maps and institutional affiliations.

\section{REFERENCES}

1. Wang, B., Wan, Y., Zheng, G. \& Hu, J. Evaluating a tap water contamination incident attributed to oil contamination by nontargeted screening strategies. Environ. Sci. Technol. 50, 2956-2963 (2016).

2. Whelton, A. J. et al. Residential tap water contamination following the freedom industries chemical spill: perceptions, water quality, and health impacts. Environ. Sci. Technol. 49, 813-823 (2015).

3. Kasprzyk-Hordern, B. \& Baker, D. R. Enantiomeric profiling of chiral drugs in wastewater and receiving waters. Environ. Sci. Technol. 46, 1681-1691 (2012).

4. Pieper, K. J., Tang, M. \& Edwards, M. A. Flint water crisis caused by interrupted corrosion control: investigating "ground zero" home. Environ. Sci. Technol. 51, 2007-2014 (2017).

5. Kumpel, E. \& Nelson, K. L. Intermittent water supply: prevalence, practice, and microbial water quality. Environ. Sci. Technol. 50, 542-553 (2016).

6. Zodrow, K. R. et al. Advanced materials, technologies, and complex systems analyses: emerging opportunities to enhance urban water security. Environ. Sci. Technol. 51, 10274-10281 (2017).

7. Elimelech, M. \& Phillip, W. A. The future of seawater desalination: energy, technology, and the environment. Science 333, 712-717 (2011).

8. Meng, F. et al. Recent advances in membrane bioreactors (MBRs): membrane fouling and membrane material. Water Res. 43, 1489-1512 (2009).

9. Werber, J. R., Osuji, C. O. \& Elimelech, M. Materials for next-generation desalination and water purification membranes. Nat. Rev. Mater. 1, 16018 (2016).

10. Fane, A. G., Wang, R. \& Hu, M. X. Synthetic membranes for water purification: status and future. Angew. Chem. Int. Ed. 54, 3368-3386 (2015).

11. Lee, A., Elam, J. W. \& Darling, S. B. Membrane materials for water purification: design, development, and application. Environ. Sci. Water Res. Technol. 2, 17-42 (2016).

12. Fritzmann, C., Löwenberg, J., Wintgens, T. \& Melin, T. State-of-the-art of reverse osmosis desalination. Desalination 216, 1-76 (2007).

13. Tong, T. \& Elimelech, M. The global rise of zero liquid discharge for wastewater management: drivers, technologies, and future directions. Environ. Sci. Technol. 50, 6846-6855 (2016).

14. Werber, J. R., Deshmukh, A. \& Elimelech, M. The critical need for increased selectivity, not increased water permeability, for desalination membranes. Environ. Sci. Technol. Lett. 3, 112-120 (2016).

15. Verbeke, R., Gómez, V. \& Vankelecom, I. F. J. Chlorine-resistance of reverse osmosis (RO) polyamide membranes. Prog. Polym. Sci. 72, 1-15 (2017).

16. Li, W.-W., Yu, H.-Q. \& Rittmann, B. E. Chemistry: reuse water pollutants. Nature 528, 29-31 (2015).

17. Larsen, T. A., Hoffmann, S., Lüthi, C., Truffer, B. \& Maurer, M. Emerging solutions to the water challenges of an urbanizing world. Science 352, 928-933 (2016).

18. Mulchandani, A. \& Westerhoff, P. Recovery opportunities for metals and energy from sewage sludges. Bioresour. Technol. 215, 215-226 (2016).
19. Mayer, B. K. et al. Total value of phosphorus recovery. Environ. Sci. Technol. 50, 6606-6620 (2016).

20. Pratt, C., Parsons, S. A., Soares, A. \& Martin, B. D. Biologically and chemically mediated adsorption and precipitation of phosphorus from wastewater. Curr. Opin. Biotechnol. 23, 890-896 (2012).

21. Hering, J. G., Waite, T. D., Luthy, R. G., Drewes, J. E. \& Sedlak, D. L. A changing framework for urban water systems. Environ. Sci. Technol. 47, 10721-10726 (2013).

22. Eggen, R. I., Hollender, J., Joss, A., Schärer, M. \& Stamm, C. Reducing the discharge of micropollutants in the aquatic environment: the benefits of upgrading wastewater treatment plants. Environ. Sci. Technol. 48, 7683-7689 (2014).

23. Altman, S. J. et al. Membrane treatment of side-stream cooling tower water for reduction of water usage. Desalination 285, 177-183 (2012).

24. Ang, W. L., Mohammad, A. W., Hilal, N. \& Leo, C. P. A review on the applicability of integrated/hybrid membrane processes in water treatment and desalination plants. Desalination 363, 2-18 (2015).

25. Stoquart, C., Servais, P., Bérubé, P. R. \& Barbeau, B. Hybrid membrane processes using activated carbon treatment for drinking water: a review. J. Membr. Sci. 411, 1-12 (2012).

26. Wu, D. et al. Design and preparation of porous polymers. Chem. Rev. 112, 3959-4015 (2012).

27. Pendergast, M. M. \& Hoek, E. M. A review of water treatment membrane nanotechnologies. Energy Environ. Sci. 4, 1946-1971 (2011).

28. Zhang, Y., Sargent, J. L., Boudouris, B. W. \& Phillip, W. A. Nanoporous membranes generated from self-assembled block polymer precursors: Quo Vadis? J. Appl. Polym. Sci. 132, 41683-41699 (2015).

29. Abetz, V. Isoporous block copolymer membranes. Macromol. Rapid Commun. 36, 10-22 (2015).

30. Mehta, A. \& Zydney, A. L. Permeability and selectivity analysis for ultrafiltration membranes. J. Membr. Sci. 249, 245-249 (2005).

31. Bates, F. S. et al. Multiblock polymers: panacea or pandora's box? Science 336, 434-440 (2012).

32. Bates, F. S. \& Fredrickson, G. H. Block copolymers-designer soft materials. Phys. Today 52, 32-38 (1999).

33. Bates, C. M. \& Bates, F. S. 50th anniversary perspective: block polymers-pure potential. Macromolecules 50, 3-22 (2017).

34. Rahman, A., Majewski, P. W., Doerk, G., Black, C. T. \& Yager, K. G. Non-native three-dimensional block copolymer morphologies. Nat. Commun. 7, 13988 (2016).

35. Phillip, W. A., O'Neill, B., Rodwogin, M., Hillmyer, M. A. \& Cussler, E. L. Selfassembled block copolymer thin films as water filtration membranes. ACS Appl. Mater. Interfaces 2, 847-853 (2010).

36. Park, S. et al. Giant gyroid and templates from high-molecular-weight block copolymer self-assembly. Sci. Rep. 6, 36326 (2016).

37. Li, L. et al. Ultrafiltration by gyroid nanoporous polymer membranes. J. Membr. Sci. 384, 126-135 (2011).

38. Yin, J. et al. Membranes with highly ordered straight nanopores by selective swelling of fast perpendicularly aligned block copolymers. ACS Nano 7, 9961-9974 (2013).

39. Wei, M., Sun, W., Shi, X., Wang, Z. \& Wang, Y. Homoporous membranes with tailored pores by soaking block copolymer/homopolymer blends in selective solvents: dissolution versus swelling. Macromolecules 49, 215-223 (2015).

40. Albert, J. N. \& Epps, T. H. Self-assembly of block copolymer thin films. Mater. Today 13, 24-33 (2010).

41. Querelle, S. E., Jackson, E. A., Cussler, E. L. \& Hillmyer, M. A. Ultrafiltration membranes with a thin poly(styrene)-b-poly(isoprene) selective layer. ACS Appl. Mater. Interfaces 5, 5044-5050 (2013).

42. Jackson, E. A., Lee, Y. \& Hillmyer, M. A. ABAC tetrablock terpolymers for tough nanoporous filtration membranes. Macromolecules 46, 1484-1491 (2013).

43. Yang, S. Y. et al. Nanoporous membranes with ultrahigh selectivity and flux for the filtration of viruses. Adv. Mater. 18, 709-712 (2006).

44. Paradiso, S. P., Delaney, K. T., García-Cervera, C. J., Ceniceros, H. D. \& Fredrickson, G. H. Block copolymer self assembly during rapid solvent evaporation: insights into cylinder growth and stability. ACS Macro Lett. 3, 16-20 (2013).

45. Hao, J. et al. Self-assembly in block copolymer thin films upon solvent evaporation: a simulation study. Macromolecules 50, 4384-4396 (2017).

46. Phillip, W. A., Hillmyer, M. A. \& Cussler, E. L. Cylinder orientation mechanism in block copolymer thin films upon solvent evaporation. Macromolecules 43, 7763-7770 (2010).

47. Ferrarese Lupi, F. et al. GISAXS analysis of the in-depth morphology of thick PSb-PMMA films. ACS Appl. Mater. Interfaces 9, 11054-11063 (2017).

48. Yang, S. Y. et al. Virus filtration membranes prepared from nanoporous block copolymers with good dimensional stability under high pressures and excellent solvent resistance. Adv. Funct. Mater. 18, 1371-1377 (2008). 
49. Phillip, W. A., Rzayev, J., Hillmyer, M. A. \& Cussler, E. L. Gas and water liquid transport through nanoporous block copolymer membranes. J. Membr. Sci. 286, 144-152 (2006).

50. Bolton, J., Bailey, T. S. \& Rzayev, J. Large pore size nanoporous materials from the self-assembly of asymmetric bottlebrush block copolymers. Nano Lett. 11, 998-1001 (2011).

51. Rzayev, J. \& Hillmyer, M. A. Nanochannel array plastics with tailored surface chemistry. J. Am. Chem. Soc. 127, 13373-13379 (2005).

52. Jackson, E. A., Lee, Y., Radlauer, M. R. \& Hillmyer, M. A. Well-ordered nanoporous aba copolymer thin films via solvent vapor annealing, homopolymer blending, and selective etching of abac tetrablock terpolymers. ACS Appl. Mater. Interfaces 7, 27331-27339 (2015).

53. Park, S. et al. Macroscopic 10-terabit-per-square-inch arrays from block copolymers with lateral order. Science 323, 1030-1033 (2009).

54. Bang, J. et al. Effect of humidity on the ordering of PEO-based copolymer thin films. Macromolecules 40, 7019-7025 (2007).

55. Posselt, D. et al. Restructuring in block copolymer thin films: in situ GISAXS investigations during solvent vapor annealing. Prog. Polym. Sci. 66, 80-115 (2017).

56. Peinemann, K.-V., Abetz, V. \& Simon, P. F. W. Asymmetric superstructure formed in a block copolymer via phase separation. Nat. Mater. 6, 992-996 (2007).

57. Rangou, S. et al. Self-organized isoporous membranes with tailored pore sizes. J. Membr. Sci. 451, 266-275 (2014).

58. Phillip, W. A. et al. Tuning structure and properties of graded triblock terpolymer-based mesoporous and hybrid films. Nano Lett. 11, 2892-2900 (2011).

59. Nunes, S. P. et al. Switchable pH-responsive polymeric membranes prepared via block copolymer micelle assembly. ACS Nano 5, 3516-3522 (2011).

60. Pendergast, M. M., Dorin, R. M., Phillip, W. A., Wiesner, U. \& Hoek, E. M. Understanding the structure and performance of self-assembled triblock terpolymer membranes. J. Membr. Sci. 444, 461-468 (2013).

61. Sargent, J. L., Hoss, D. J., Phillip, W. A. \& Boudouris, B. W. Solution self-assembly behavior of $\mathrm{A}-\mathrm{B}-\mathrm{C}$ triblock polymers and the implications for nanoporous membrane fabrication. J. Appl. Polym. Sci. https://doi.org/10.1002/app.45531 (2017).

62. Radjabian, M., Abetz, C., Fischer, B., Meyer, A. \& Abetz, V. Influence of solvent on the structure of an amphiphilic block copolymer in solution and in formation of an integral asymmetric membrane. ACS Appl. Mater. Interfaces 9, 31224-31234 (2017).

63. Marques, D. S. et al. Self-assembly in casting solutions of block copolymer membranes. Soft Matter 9, 5557-5564 (2013).

64. Sutisna, B. et al. Design of block copolymer membranes using segregation strength trend lines. Mol. Syst. Des. Eng. 1, 278-289 (2016).

65. Nunes, S. P. Block copolymer membranes for aqueous solution applications. Macromolecules 49, 2905-2916 (2016).

66. Oss-Ronen, L. et al. Characterization of block copolymer self-assembly: from solution to nanoporous membranes. Macromolecules 45, 9631-9642 (2012).

67. Dorin, R. M. et al. Solution small-angle $x$-ray scattering as a screening and predictive tool in the fabrication of asymmetric block copolymer membranes. ACS Macro Lett. 1, 614-617 (2012).

68. Marques, D. S. et al. Time-resolved GISAXS and cryo-microscopy characterization of block copolymer membrane formation. Polymer 55, 1327-1332 (2014).

69. Stegelmeier, C. et al. Evaporation-induced block copolymer self-assembly into membranes studied by in situ synchrotron SAXS. Macromolecules 48, 1524-1530 (2015).

70. Gu, Y., Dorin, R. M., Tan, K. W., Smilgies, D.-M. \& Wiesner, U. In situ study of evaporation-induced surface structure evolution in asymmetric triblock terpolymer membranes. Macromolecules 49, 4195-4201 (2016).

71. Choi, S.-H., Bates, F. S. \& Lodge, T. P. Small-angle x-ray scattering of concentration dependent structures in block copolymer solutions. Macromolecules 47, 7978-7986 (2014)

72. Li, Y. M. et al. Effect of humidity on surface structure and permeation of triblock terpolymer derived SNIPS membranes. Polymer 126, 368-375 (2017)

73. Zhang, Q., Li, Y. M., Gu, Y., Dorin, R. M. \& Wiesner, U. Tuning substructure and properties of supported asymmetric triblock terpolymer membranes. Polymer 107, 398-405 (2016).

74. Guillen, G. R., Pan, Y., Li, M. \& Hoek, E. M. Preparation and characterization of membranes formed by nonsolvent induced phase separation: a review. Ind. Eng. Chem. Res. 50, 3798-3817 (2011)

75. Gu, Y., Dorin, R. M. \& Wiesner, U. Asymmetric organic-inorganic hybrid membrane formation via block copolymer-nanoparticle co-assembly. Nano Lett. 13 5323-5328 (2013).

76. Gu, Y. \& Wiesner, U. Tailoring pore size of graded mesoporous block copolymer membranes: moving from ultrafiltration toward nanofiltration. Macromolecules 48, 6153-6159 (2015).
77. Hahn, J., Clodt, J. I., Abetz, C., Filiz, V. \& Abetz, V. Thin isoporous block copolymer membranes: it is all about the process. ACS Appl. Mater. Interfaces 7 , 21130-21137 (2015).

78. Zhang, Y., Mulvenna, R. A., Boudouris, B. W. \& Phillip, W. A. Nanomanufacturing of high-performance hollow fiber nanofiltration membranes by coating uniform block polymer films from solution. J. Mater. Chem. A 5, 3358-3370 (2017).

79. Dorin, R. M. et al. Designing block copolymer architectures for targeted membrane performance. Polymer 55, 347-353 (2014).

80. Kim, S. H., Kwak, S.-Y. \& Suzuki, T. Positron annihilation spectroscopic evidence to demonstrate the flux-enhancement mechanism in morphology-controlled Thin-Film-Composite (TFC) membrane. Environ. Sci. Technol. 39, 1764-1770 (2005).

81. Shintani, T., Shimazu, A., Yahagi, S. \& Matsuyama, H. Characterization of methylsubstituted polyamides used for reverse osmosis membranes by positron annihilation lifetime spectroscopy and MD simulation. J. Appl. Polym. Sci. 113, 1757-1762 (2009).

82. Wei, T. et al. Aromatic polyamide reverse-osmosis membrane: an atomistic molecular dynamics simulation. J. Phys. Chem. B 120, 10311-10318 (2016).

83. Harder, E., Walters, D. E., Bodnar, Y. D., Faibish, R. S. \& Roux, B. Molecular dynamics study of a polymeric reverse osmosis membrane. J. Phys. Chem. B 113 10177-10182 (2009).

84. Bates, F. S. \& Fredrickson, G. H. Block copolymer thermodynamics: theory and experiment. Annu. Rev. Phys. Chem. 41, 525-557 (1990).

85. van Genabeek, B. et al. Synthesis and self-assembly of discrete dimethylsiloxane-lactic acid diblock co-oligomers: the dononacontamer and its shorter homologues. J. Am. Chem. Soc. 138, 4210-4218 (2016).

86. Pitet, L. M. et al. Well-organized dense arrays of nanodomains in thin films of poly (dimethylsiloxane)-b-poly(lactide) diblock copolymers. Macromolecules 46, 8289-8295 (2013).

87. Cushen, J. D. et al. Oligosaccharide/silicon-containing block copolymers with 5 $\mathrm{nm}$ features for lithographic applications. ACS Nano 6, 3424-3433 (2012).

88. Durand, W. J. et al. Design of high- $\chi$ block copolymers for lithography. J. Polym. Sci., Part A: Polym. Chem. 53, 344-352 (2015).

89. Sing, C. E., Zwanikken, J. W. \& De La Cruz, M. O. Electrostatic control of block copolymer morphology. Nat. Mater. 13, 694-698 (2014).

90. Sinturel, C., Bates, F. S. \& Hillmyer, M. A. High X-low $N$ block polymers: how far can we go? ACS Macro Lett. 4, 1044-1050 (2015).

91. Feng, X., Kawabata, K., Kaufman, G., Elimelech, M. \& Osuji, C. O. Highly selective vertically aligned nanopores in sustainably derived polymer membranes by molecular templating. ACS Nano 11, 3911-3921 (2017).

92. Mulvenna, R. A. et al. Tunable nanoporous membranes with chemically-tailored pore walls from triblock polymer templates. J. Membr. Sci. 470, 246-256 (2014).

93. Zhang, Y., Mulvenna, R. A., Qu, S., Boudouris, B. W. \& Phillip, W. A. Block polymer membranes functionalized with nanoconfined polyelectrolyte brushes achieve sub-nanometer selectivity. ACS Macro Lett. 6, 726-732 (2017).

94. $\mathrm{Yu}, \mathrm{H}$. et al. Self-assembled asymmetric block copolymer membranes: bridging the gap from ultra-to nanofiltration. Angew. Chem., Int. Ed. 54, 13937-13941 (2015).

95. Wang, Y., He, Z., Gupta, K. M., Shi, Q. \& Lu, R. Molecular dynamics study on water desalination through functionalized nanoporous graphene. Carbon 116, 120-127 (2017)

96. Corry, B. Designing carbon nanotube membranes for efficient water desalination. J. Phys. Chem. B 112, 1427-1434 (2008).

97. Thomas, M. \& Corry, B. A computational assessment of the permeability and salt rejection of carbon nanotube membranes and their application to water desalination. Philos. Trans. R. Soc., A 374, 1-20 (2016).

98. Miller, D. J., Dreyer, D. R., Bielawski, C. W., Paul, D. R. \& Freeman, B. D. Surface modification of water purification membranes. Angew. Chem., Int. Ed. 56, 4662-4711 (2017).

99. Gamys, C. G., Schumers, J. M., Mugemana, C., Fustin, C. A. \& Gohy, J. F. Porefunctionalized nanoporous materials derived from block copolymers. Macromol. Rapid Commun. 34, 962-982 (2013).

100. Vankelecom, I. F. Polymeric membranes in catalytic reactors. Chem. Rev. 102, 3779-3810 (2002)

101. Dotzauer, D. M., Dai, J., Sun, L. \& Bruening, M. L. Catalytic membranes prepared using layer-by-layer adsorption of polyelectrolyte/metal nanoparticle films in porous supports. Nano Lett. 6, 2268-2272 (2006).

102. Chitpong, N. \& Husson, S. M. Polyacid functionalized cellulose nanofiber membranes for removal of heavy metals from impaired waters. J. Membr. Sci. 523, 418-429 (2017).

103. Smuleac, V., Butterfield, D. A., Sikdar, S. K., Varma, R. S. \& Bhattacharyya, D. Polythiol-functionalized alumina membranes for mercury capture. J. Membr. Sci. 251, 169-178 (2005).

104. Wei, Y. M., Li, Y., Yang, C. \& Cussler, E. L. More effective membrane chromatography. AlChE J. 61, 3871-3878 (2015). 
105. Schisla, D. K., Ding, H., Carr, P. W. \& Cussler, E. Polydisperse tube diameters compromise multiple open tubular chromatography. AIChE J. 39, 946-953 (1993).

106. Madhavan, P., Hong, P.-Y., Sougrat, R. \& Nunes, S. P. Silver-enhanced block copolymer membranes with biocidal activity. ACS Appl. Mater. Interfaces 6 , 18497-18501 (2014).

107. Hilke, R. et al. Block copolymer hollow fiber membranes with catalytic activity and pH-response. ACS Appl. Mater. Interfaces 5, 7001-7006 (2013).

108. Yu, H., Qiu, X., Nunes, S. P. \& Peinemann, K. V. Self-assembled isoporous block copolymer membranes with tuned pore sizes. Angew. Chem. Int. Ed. 53, 10072-10076 (2014).

109. Rao, J. et al. Self-assembly of an interacting binary blend of diblock copolymers in thin films: a potential route to porous materials with reactive nanochannel chemistry. Soft Matter 10, 5755-5762 (2014).

110. Li, Y. M., Srinivasan, D., Vaidya, P., Gu, Y. \& Wiesner, U. Asymmetric membranes from two chemically distinct triblock terpolymers blended during standard membrane fabrication. Macromol. Rapid Commun. 37, 1689-1693 (2016).

111. Gamys, C. G., Vlad, A., Bertrand, O. \& Gohy, J. F. Functionalized nanoporous thin films from blends of block copolymers and homopolymers interacting via hydrogen bonding. Macromol. Chem. Phys. 213, 2075-2080 (2012).

112. Jung, $A$. et al. Formation of integral asymmetric membranes of $A B$ diblock and ABC triblock copolymers by phase inversion. Macromol. Rapid Commun. 34, 610-615 (2013)

113. Clodt, J. I. et al. Double stimuli-responsive isoporous membranes via postmodification of $\mathrm{pH}$-sensitive self-assembled diblock copolymer membranes. Adv. Funct. Mater. 23, 731-738 (2013).

114. Keskin, D., Clodt, J. I., Hahn, J., Abetz, V. \& Filiz, V. Postmodification of PS-b-P4VP diblock copolymer membranes by ARGET ATRP. Langmuir 30, 8907-8914 (2014).

115. Shevate, R., Kumar, M., Karunakaran, M., Hedhili, M. N. \& Peinemann, K.-V. Polydopamine/Cysteine surface modified isoporous membranes with selfcleaning properties. J. Membr. Sci. 529, 185-194 (2017).

116. Peng, Q., Tseng, Y.-C., Darling, S. B. \& Elam, J. W. A route to nanoscopic materials via sequential infiltration synthesis on block copolymer templates. ACS Nano $\mathbf{5}$, 4600-4606 (2011).

117. Cowman, C. D. et al. Multicomponent nanomaterials with complex networked architectures from orthogonal degradation and binary metal backfilling in $A B C$ triblock terpolymers. J. Am. Chem. Soc. 137, 6026-6033 (2015).

118. Gu, Y., Werner, J. G., Dorin, R. M., Robbins, S. W. \& Wiesner, U. Graded porous inorganic materials derived from self-assembled block copolymer templates. Nanoscale 7, 5826-5834 (2015).

119. Susca, E. M. et al. Self-assembled gyroidal mesoporous polymer-derived high temperature ceramic monoliths. Chem. Mater. 28, 2131-2137 (2016).

120. Zhou, C. et al. Fabrication of nanoporous alumina ultrafiltration membrane with tunable pore size using block copolymer templates. Adv. Funct. Mater. 27, 1701756 (2017).

121. Dorin, R. M., Sai, H. \& Wiesner, U. Hierarchically porous materials from block copolymers. Chem. Mater. 26, 339-347 (2014).

122. Qiu, X. et al. Selective separation of similarly sized proteins with tunable nanoporous block copolymer membranes. ACS Nano 7, 768-776 (2012).

123. Tripathi, B. P., Dubey, N. C., Choudhury, S., Simon, F. \& Stamm, M. Antifouling and antibiofouling $\mathrm{pH}$ responsive block copolymer based membranes by selective surface modification. J. Mater. Chem. B 1, 3397-3409 (2013).

124. Weidman, J. L., Mulvenna, R. A., Boudouris, B. W. \& Phillip, W. A. Nanostructured membranes from triblock polymer precursors as high capacity copper adsorbents. Langmuir 31, 11113-11123 (2015).

125. Weidman, J. L., Mulvenna, R. A., Boudouris, B. W. \& Phillip, W. A. Nanoporous block polymer thin films functionalized with bio-inspired ligands for the efficient capture of heavy metal ions from water. ACS Appl. Mater. Interfaces 9, 19152-19160 (2017)

126. Oleske, K. W. et al. Block copolymer directed nanostructured surfaces as templates for confined surface reactions. Macromolecules 50, 542-549 (2017).

127. Wei, X. et al. Synthesis and morphology investigations of a novel alkyne-functionalized diblock copolymer. J. Polym. Sci., Part B. 51, 78-85 (2013).

128. Zhang, Q. et al. Dynamically responsive multifunctional asymmetric triblock terpolymer membranes with intrinsic binding sites for covalent molecule attachment. Chem. Mater. 28, 3870-3876 (2016).
129. Mulder, D. J. et al. Fabrication and post-modification of nanoporous liquid crystalline networks via dynamic covalent chemistry. Chem. Mater. 29, 6601-6605 (2017).

130. Bailey, T. S., Rzayev, J. \& Hillmyer, M. A. Routes to alkene and epoxide functionalized nanoporous materials from poly(styrene-b-isoprene-b-lactide) triblock copolymers. Macromolecules 39, 8772-8781 (2006).

131. Choi, J. W. et al. Self-assembly and post-fabrication functionalization of microphase separated thin films of a reactive azlactone-containing block copolymer. Macromolecules 49, 8177-8186 (2016).

132. McVerry, B. T. et al. Scalable antifouling reverse osmosis membranes utilizing perfluorophenyl azide photochemistry. Macromol. Rapid Commun. 35, 1528-1533 (2014).

133. Huang, X., Marsh, K. L., McVerry, B. T., Hoek, E. M. \& Kaner, R. B. Low-fouling antibacterial reverse osmosis membranes via surface grafting of graphene oxide. ACS Appl. Mater. Interfaces 8, 14334-14338 (2016).

134. Barry, E., McBride, S. P., Jaeger, H. M. \& Lin, X.-M. Ion transport controlled by nanoparticle-functionalized membranes. Nat. Commun. 5, 5847-5854 (2014).

135. Qu, S., Dilenschneider, T. \& Phillip, W. A. Preparation of chemically-tailored copolymer membranes with tunable ion transport properties. ACS Appl. Mater. Interfaces 7, 19746-19754 (2015).

136. Qu, S. et al. Copolymer nanofilters with charge-patterned domains for enhanced electrolyte transport. Chem. Mater. 29, 762-772 (2016).

137. Rathee, V. S., Qu, S., Phillip, W. A. \& Whitmer, J. K. A coarse-grained thermodynamic model for the predictive engineering of valence-selective membranes. Mol. Syst. Des. Eng. 1, 301-312 (2016).

138. Das, S. et al. Extracting uranium from seawater: promising AF series adsorbents. Ind. Eng. Chem. Res. 55, 4110-4117 (2015).

139. Das, S. et al. Extracting uranium from seawater: promising Al series adsorbents. Ind. Eng. Chem. Res. 55, 4103-4109 (2015).

140. Ghosh, S. \& Chakrabarti, R. Molecular dynamics simulation elucidates the preferential binding affinity of sodium and tetramethylammonium ions for tetrameric Nafion unit under aqueous conditions. RSC Adv. 6, 97961-97968 (2016).

141. Alkhudhiri, A., Darwish, N. \& Hilal, N. Membrane distillation: a comprehensive review. Desalination 287, 2-18 (2012).

142. Straub, A. P., Yip, N. Y., Lin, S., Lee, J. \& Elimelech, M. Harvesting low-grade heat energy using thermo-osmotic vapour transport through nanoporous membranes. Nat. Energy 1, 16090 (2016).

143. Hughes, Z. E. \& Gale, J. D. Molecular dynamics simulations of the interactions of potential foulant molecules and a reverse osmosis membrane. J. Mater. Chem. 22, 175-184 (2012)

144. Rana, D. \& Matsuura, T. Surface modifications for antifouling membranes. Chem. Rev. 110, 2448-2471 (2010).

145. Jiang, S. \& Cao, Z. Ultralow-fouling, functionalizable, and hydrolyzable zwitterionic materials and their derivatives for biological applications. Adv. Mater. 22, 920-932 (2010).

146. Ostuni, E., Chapman, R. G., Holmlin, R. E., Takayama, S. \& Whitesides, G. M. A survey of structure-property relationships of surfaces that resist the adsorption of protein. Langmuir 17, 5605-5620 (2001).

147. Zhou, M. et al. High throughput discovery of new fouling-resistant surfaces. J. Mater. Chem. 21, 693-704 (2011).

(i) Open Access This article is licensed under a Creative Commons Attribution 4.0 International License, which permits use, sharing, adaptation, distribution and reproduction in any medium or format, as long as you give appropriate credit to the original author(s) and the source, provide a link to the Creative Commons license, and indicate if changes were made. The images or other third party material in this article are included in the article's Creative Commons license, unless indicated otherwise in a credit line to the material. If material is not included in the article's Creative Commons license and your intended use is not permitted by statutory regulation or exceeds the permitted use, you will need to obtain permission directly from the copyright holder. To view a copy of this license, visit http://creativecommons. org/licenses/by/4.0/.

(c) The Author(s) 2018 